\title{
SKINNY-AEAD and SKINNY-Hash
}

\author{
Christof Beierle ${ }^{1 \S}$, Jérémy Jean ${ }^{2}$, Stefan Kölbl ${ }^{3}$, Gregor Leander ${ }^{1}$, \\ Amir Moradi ${ }^{1}$, Thomas Peyrin ${ }^{4}$, Yu Sasaki $^{5}$, Pascal Sasdrich ${ }^{1}$ and \\ Siang Meng $\operatorname{Sim}^{4}$ \\ 1 Ruhr-Universität Bochum, Bochum, Germany \\ ${ }^{2}$ Agence nationale de la sécurité des systèmes d'information (ANSSI), Paris, France \\ ${ }^{3}$ Independent ${ }^{\|}$ \\ ${ }^{4}$ School of Physical and Mathematical Sciences, Nanyang Technological University, Jurong West, \\ Singapore \\ ${ }^{5}$ NTT Secure Platform Laboratories, Tokyo, Japan \\ skinny@googlegroups.com
}

\begin{abstract}
We present the family of authenticated encryption schemes SKINNY-AEAD and the family of hashing schemes SKINNY-Hash. All of the schemes employ a member of the SKINNY family of tweakable block ciphers, which was presented at CRYPTO 2016, as the underlying primitive. In particular, for authenticated encryption, we show how to instantiate members of SKINNY in the Deoxys-I-like $\Theta C B 3$ framework to fulfill the submission requirements of the NIST lightweight cryptography standardization process. For hashing, we use SKINNY to build a function with larger internal state and employ it in a sponge construction. To highlight the extensive amount of third-party analysis that SKINNY obtained since its publication, we briefly survey the existing cryptanalysis results for SKINNY-128-256 and SKINNY-128-384 as of February 2020. In the last part of the paper, we provide a variety of ASIC implementations of our schemes and propose new simple SKINNY-AEAD and SKINNY-Hash variants with a reduced number of rounds while maintaining a very comfortable security margin.
\end{abstract}

Keywords: SKINNY · Tweakable block cipher · Authenticated encryption · Hash function · NIST · Lightweight cryptography

\section{Introduction}

SKINNY is a family of lightweight tweakable block ciphers proposed at CRYPTO 2016 $\left[\mathrm{BJK}^{+} 16 \mathrm{a}\right]$. We specify how to provide the authenticated encryption and hashing functionalities, with the parameters as required in the NIST lightweight cryptography standardization process, by using SKINNY as a base primitive.

Parts of this work is based on already published results. The new contributions can be summarized as follows: We show how members of the SKINNY family of tweakable block ciphers can be instantiated in the $\Theta$ CB3 framework [KR11] in order to fulfill the requirements for the NIST lightweight cryptography standardization process ${ }^{1}$ (see also [Nat18]) and provide 6 members of a new family of AEAD schemes, called SKINNY-AEAD. We further

\footnotetext{
$\S$ Part of the work of Christof Beierle was performed while he was affiliated with the University of Luxembourg

\Part of the work of Pascal Sasdrich was performed while he was affiliated with Rambus Cryptoraphy, the Netherlands

$\|$ Stefan Kölbl is now working at Google.

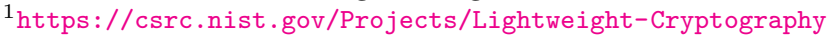


use members of the SKINNY family to construct functions with state sizes of 256 and 512 bit, which can be used in a sponge-based hashing mode, and define two members of a new family of hash functions, called SKINNY-Hash. We provide several ASIC implementations for all our SKINNY-AEAD and SKINNY-Hash members. To stress the extensive amount of existing cryptanalysis of the SKINNY family of tweakable block ciphers, we provide a survey on the external cryptanalysis of SKINNY-128-256 and SKINNY-128-384 as of February 2020 .

\subsection{SKINNY-AEAD}

In short, SKINNY-AEAD uses a mode following the general $\Theta C B 3$ framework, instantiated with SKINNY-128-384. The fact that SKINNY is a beyond birthday-bound secure tweakable block cipher enables to achieve the provable security providing full security in the noncerespecting setting. A similar mode was also employed in the third-round CAESAR candidate Deoxys-I [JNPS16]. Our primary design takes a 128-bit key, a 128-bit nonce, and an associated data and a message of up to $2^{64} \times 16$ bytes. It then outputs a ciphertext of the same length as the plaintext and a 128-bit tag. We also specify other members of this family to support any combination of $n_{\ell^{-}}$and $t_{\ell^{-}}$-bit nonces and tags, respectively, where $n_{\ell} \in\{96,128\}$ and $t_{\ell} \in\{64,128\}$.

Moreover, we also specify the lightweight version instantiated with SKINNY-128-256. This design is motivated from the observation that the submission requirement to support $2^{50}$ input bytes might be unnecessary for several of the use cases of the lightweight cryptography. This family consists of two members that take a 128-bit key, a 96-bit nonce, and an associated data and a message of up to $2^{28}$ bytes as input and produce the ciphertext and a $t_{\ell}$-bit tag, where $t_{\ell} \in\{64,128\}$. Because of the restriction of the maximum number of input message bytes, this family does not satisfy the submission requirement to support input messages of up to $2^{50}$ bytes, yet provides even smaller and faster AEAD schemes.

\subsection{SKINNY-Hash}

SKINNY-Hash consists of two members of the hash function schemes that adopt the wellknown sponge construction. Our primary member uses a 384-bit to 384-bit function built with SKINNY-128-384 to provide a 128-bit secure hash function and the secondary member uses a 256-bit to 256-bit function built with SKINNY-128-256 to provide a 112-bit secure hash function.

\subsection{Features}

Before going into the specifications, we briefly summarize the main features of our design.

- Well-understood design and high security margin. The SKINNY family of tweakable block ciphers was designed as a solid Substitution-Permutation network (SPN) having a well-analyzed security bound against the most fundamental cryptanalytic approaches: differential cryptanalysis [BS90] and linear cryptanalysis [Mat93]. In addition, SKINNY receives a lot of security analysis by third-party researchers, which demonstrates its strong resistance against cryptanalysis. The cipher can basically be understood as a tweakable version of a tailored AES which omits components not strictly necessary for the security or substitutes them by more lightweight choices. Therefore, similar cryptanalytic approaches as for AES can be applied. However, opposed to AES, the TWEAKEY framework allows to derive strong security arguments in the related-key, related-tweak setting for SKINNY. Moreover, SKINNY offers a high security margin. As of February 2020, based on our own cryptanalysis and the 
extensive external cryptanalysis since its publication, SKINNY-128-384 offers 28 (out of 56) rounds, and SKINNY-128-256 offers 25 (out of 48) rounds of security margin in the related-tweakey setting.

- Security proofs by a modular approach. The security of the authenticated encryption schemes and hash functions are directly inherited from the well-known and widely-applied modes of operation used in our design. Indeed, SKINNY-AEAD relies on the proofs of the $\Theta C B 3$ mode, while for SKINNY-Hash we rely on the provable security of the sponge framework. The security of our schemes can thus be reduced to the ideal behavior of the underlying primitives SKINNY-128-384 and SKINNY-128-256.

- Beyond-birthday-bound security. By using a tweakable block cipher directly constructed by the TWEAKEY framework, we obtain beyond-birthday-bound security which allows to efficiently exploit the whole state. This is different to modes based on OCB, which only offers security up to the birthday bound. Such modes would require larger internal states to achieve the same security level.

Note that, however, OCB is birthday-bound secure based on the SPRP assumption while $\Theta C B 3$ is beyond-birthday-bound secure based on the STPRP assumption. Clearly, an oracle to a tweakable block cipher gives more freedom to the attacker than an oracle to a classical block cipher.

- Efficient protection against side-channel attacks. Thanks to the structured Sbox of SKINNY, which is an iteration of a quadratic permutation, its Threshold Implementation [NRS11] (a provably-secure countermeasure against side-channel analysis attacks) can be efficiently made. This helps us to efficiently integrate sidechannel countermeasures into various implementations of SKINNY with minimum number of shares and limited number of fresh randomness, both affecting the area overhead of the resulting design.

- General-purpose lightweight schemes. When designing a lightweight encryption or hashing scheme, several use cases must be taken in account. While area-optimized implementations are important for some very constrained applications, throughput or throughput-over-area optimized implementations are also very relevant. Actually, looking at recently introduced efficiency measurements (the FOAM value - Figure Of Adversarial Merit [KPPY14]), one can see that our design choices are good for many types of implementations, which is exactly what makes a good general-purpose lightweight encryption scheme.

- Efficiency for short messages. Our algorithms are efficient for short messages. For authenticated encryption, the main reason is because the design is based on a tweakable block cipher, which allows to avoid any precomputation (like in OCB, AES-GCM, etc.). In particular, the first 128-bit message block is handled directly and by taking in account the tag generation, one needs only $m+1$ internal calls to the tweakable block cipher to process messages of $m$ blocks of 128 bits each (if there is no associated data).

Our primary member for hashing requires at most $3(m+2)$ calls to the tweakable block cipher for producing a 256-bit digest for a message of $m$ blocks of 128 bits each.

- Parallelizable mode. Our AEAD schemes are fully parallelizable as they are based on the $\Theta$ CB3 mode, which employs independent calls to the tweakable block cipher.

- Flexibility. Our AEAD design allows for smooth parameter handling. We define specific parameter sets to achieve the NIST requirements, but any user can in 
principle choose its own separation into nonce, key and block counter by adapting the key and tweak sizes at his/her convenience. This flexibility comes from the unified vision of the key and tweak material brought by the TWEAKEY framework. In a nutshell, one implementation of the underlying cipher is sufficient to support all versions with different key and tweak sizes (which sum up to the same size).

\section{Specification}

By $\|$ we denote the concatenation of bitstrings. Given a bitstring $B$, we denote by $B^{j}$ the $j$-times concatenation of $B$, where $B^{0}$ is defined to be the empty string $\epsilon$. For instance $0 \| 10^{3}=010^{3}=01000$ and $(10)^{3}=101010$. We denote the length of a string $B$ in bits by $|B|$, where $|\epsilon|=0$.

\subsection{Parameter Sets}

\subsubsection{AEAD}

In a nutshell, our AEAD scheme adopts a mode that can be described in the $\Theta C B 3$ framework [KR11] by using either SKINNY-128-384 or SKINNY-128-256 as the underlying tweakable block cipher.

Our primary member instantiates the $\Theta \mathrm{CB} 3$ framework with the tweakable block cipher SKINNY-128-384 used with 128-bit keys, 128-bit nonces and which produces 128-bit authentication tags. Along with this primary AEAD scheme, we propose three additional ones that extend the possible parameters and allow users to choose between two nonce sizes (96 bits or 128 bits), and two tag sizes (128 bits or 64 bits). All of them are consistent with NIST's requirements.

We also specify two secondary options that are designed for processing short inputs. Those are based on a second tweakable block cipher, namely SKINNY-128-256. The nonce size is fixed to 96 bits, while users can choose between two tag sizes: 128 or 64 bits. The maximum number of message blocks that can be processed with SKINNY-128-256-based members is limited to $2^{28}$ bytes. Users need to be careful about its usage because these two algorithms do not meet NIST's requirements to support input messages of up to $2^{50}$ bytes.

\subsubsection{Hashing}

Overall, the SKINNY-Hash family contains two function-based sponge constructions (see [BDPA11]), in which the underlying functions are built from the SKINNY-128-384 and SKINNY-128-256 tweakable block ciphers. Both members, denoted SKINNY-tk3-Hash and SKINNY-tk2-Hash, process input messages of arbitrary length and output a 256-bit digest.

A list of our proposed AEAD schemes (members M1 to M6), together with the two hashing algorithms is provided in Table 1. For comparisons, we pair the AEAD members M1, M2, M3 and M4 with the hashing algorithm SKINNY-tk3-Hash and the AEAD members M5 and M6 with SKINNY-tk2-Hash as the constructions in the respective pairs are based on the same variant of the SKINNY tweakable block cipher.

\subsection{SKINNY-128-256 and SKINNY-128-384}

We already published the SKINNY family of tweakable block ciphers in 2016 in [BJK ${ }^{+} 16 \mathrm{a}$ ]. For the sake of completeness, we provide the specifications of the two members of the SKINNY family that are relevant for our constructions, namely SKINNY-128-256 and SKINNY-128-384. 
Table 1: Our proposed AEAD schemes and hashing algorithms.

\begin{tabular}{|c|c|c|c|c|c|c|c|}
\hline & $\begin{array}{c}\text { Block Cipher } \\
\text { underlying primitive }\end{array}$ & $\begin{array}{c}\text { Nonce } \\
\text { bits }\end{array}$ & $\begin{array}{l}\text { Tag } \\
\text { bits }\end{array}$ & $\begin{array}{c}\text { Key } \\
\text { bits }\end{array}$ & $\begin{array}{c}\text { Hash Function } \\
\text { type }\end{array}$ & $\begin{array}{c}\text { Rate } \\
\text { bits }\end{array}$ & $\begin{array}{c}\text { Capacity } \\
\text { bits }\end{array}$ \\
\hline $\mathrm{M} 1 \dagger$ & SKINNY-128-384 & 128 & 128 & 128 & \multirow{4}{*}{ 384-bit sponge } & \multirow{4}{*}{128} & \multirow{4}{*}{256} \\
\hline M2 & SKINNY-128-384 & 96 & 128 & 128 & & & \\
\hline M3 & SKINNY-128-384 & 128 & 64 & 128 & & & \\
\hline M4 & SKINNY-128-384 & 96 & 64 & 128 & & & \\
\hline M5 * & SKINNY-128-256 & 96 & 128 & 128 & \multirow{2}{*}{ 256-bit sponge } & \multirow{2}{*}{32} & \multirow{2}{*}{224} \\
\hline $\mathrm{M}^{*}$ & SKINNY-128-256 & 96 & 64 & 128 & & & \\
\hline
\end{tabular}

The tweakable block ciphers SKINNY-128-256 and SKINNY-128-384 both have a block size of $n=128$ bit and the internal state is viewed as a $4 \times 4$ square array of cells, where each cell contains a byte. We denote $I S_{i, j}$ the cell of the internal state located at Row $i$ and Column $j$ (counting starts from 0 ). One can also view this $4 \times 4$ square array of cells as a vector of cells by concatenating the rows. Thus, we denote with a single subscript $I S_{i}$ the cell of the internal state located at Position $i$ in this vector (counting starts from 0) and we have that $I S_{i, j}=I S_{4 \cdot i+j}$.

The ciphers follow the TWEAKEY framework from [JNP14] and therefore take a tweakey input - instead of a key only - without any distinction between key and tweak input.

The two tweakable block ciphers SKINNY-128-256 and SKINNY-128-384 mainly differ in the size of the tweakey input: they respectively process $2 n=256$ or $3 n=384$ tweakey bits. The tweakey state is also viewed as a collection of two (resp., three) $4 \times 4$ square arrays of cells of 8 bits each. We denote these arrays $T K 1$ and $T K 2$ for SKINNY-128-256 and $T K 1, T K 2$ and $T K 3$ for SKINNY-128-384. Moreover, we denote $T K z_{i, j}$ the cell of the tweakey state located at Row $i$ and Column $j$ of the $z$-th cell array. As for the internal state, we extend this notation to a vector view with a single subscript: $T K 1_{i}, T K 2_{i}$ and $T K 3_{i}$.

We now give the structural specifications of the ciphers.

\subsubsection{Initialization}

The ciphers receive a plaintext $m=m_{0}\left\|m_{1}\right\| \cdots\left\|m_{14}\right\| m_{15}$, where the $m_{i}$ are 8-bit words. The initialization of the ciphers' internal state are performed by simply setting $I S_{i}=m_{i}$ for $0 \leq i \leq 15$, i.e.,

$$
I S=\left[\begin{array}{cccc}
m_{0} & m_{1} & m_{2} & m_{3} \\
m_{4} & m_{5} & m_{6} & m_{7} \\
m_{8} & m_{9} & m_{10} & m_{11} \\
m_{12} & m_{13} & m_{14} & m_{15}
\end{array}\right]
$$

Note that the state is loaded row-wise rather than in the column-wise fashion as done for example in the AES. This is a more hardware-friendly choice, as pointed out in $\left[\mathrm{MPL}^{+} 11\right]$.

The ciphers receive a tweakey input $t k=t k_{0}\left\|t k_{1}\right\| \cdots \| t k_{31}\left(\right.$ resp., $\left.t k=t k_{0}\|\cdots\| t k_{47}\right)$, where the $t k_{i}$ are 8-bit words. The initialization of the cipher's tweakey state is performed 
by simply setting for $0 \leq i \leq 15$ :

$$
\begin{array}{rl}
\text { For } 2 n \text {-bit tweakey: } & \text { For } 3 n \text {-bit tweakey: } \\
T K 1_{i}=t k_{i} & T K 1_{i}=t k_{i} \\
T K 2_{i}=t k_{16+i} & T K 2_{i}=t k_{16+i} \\
& T K 3_{i}=t k_{32+i}
\end{array}
$$

Note that the tweakey states are also loaded row-wise.

\subsubsection{Round Function}

One encryption round of SKINNY is composed of five operations in the following order: SubCells, AddConstants, AddRoundTweakey, ShiftRows and MixColumns (see illustration in Figure 1). The number $r$ of rounds to perform during encryption depends on the tweakey

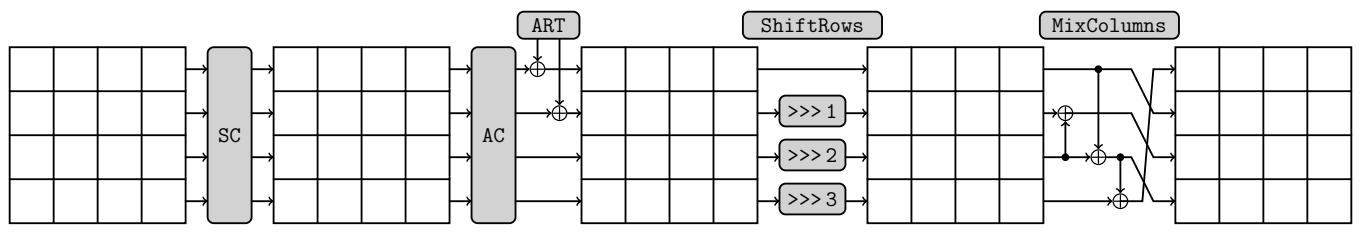

Figure 1: The SKINNY round function applies five different transformations: SubCells (SC), AddConstants (AC), AddRoundTweakey (ART), ShiftRows (SR) and MixColumns (MC).

size. In particular, SKINNY-128-256 applies $r=48$ and SKINNY-128-384 applies $r=56$ rounds. Note that no whitening key is used.

SubCells. An 8-bit Sbox $\mathcal{S}_{8}$ is applied to every cell of the ciphers internal state. Its design is simple and inspired by the PICCOLO Sbox $\left[\mathrm{SIH}^{+} 11\right]$.

If $x_{0}, \ldots, x_{7}$ represent the eight input bits of the Sbox ( $x_{0}$ being the least significant bit), it basically applies the below transformation on the 8-bit state:

$\left(x_{7}, x_{6}, x_{5}, x_{4}, x_{3}, x_{2}, x_{1}, x_{0}\right) \rightarrow\left(x_{7}, x_{6}, x_{5}, x_{4} \oplus\left(\overline{x_{7} \vee x_{6}}\right), x_{3}, x_{2}, x_{1}, x_{0} \oplus\left(\overline{x_{3} \vee x_{2}}\right)\right)$,

followed by the bit permutation

$$
\left(x_{7}, x_{6}, x_{5}, x_{4}, x_{3}, x_{2}, x_{1}, x_{0}\right) \longrightarrow\left(x_{2}, x_{1}, x_{7}, x_{6}, x_{4}, x_{0}, x_{3}, x_{5}\right),
$$

repeating this process four times, except for the last iteration where there is just a bit swap between $x_{1}$ and $x_{2}$. Besides, we provide in Appendix A the table of $\mathcal{S}_{8}$ and its inverse in hexadecimal notations.

AddConstants. A 6-bit affine LFSR, whose state is denoted $\left(\mathrm{rc}_{5}, \mathrm{rc}_{4}, \mathrm{rc}_{3}, \mathrm{rc}_{2}, \mathrm{rc}_{1}, \mathrm{rc}_{0}\right)$ (with $\mathrm{rc}_{0}$ being the least significant bit), is used to generate round constants. Its update function is defined as:

$$
\left(\mathrm{rc}_{5}|| \mathrm{rc}_{4}\left\|\mathrm{rc}_{3}\right\| \mathrm{rc}_{2}\left\|\mathrm{rc}_{1}\right\| \mathrm{rc}_{0}\right) \rightarrow\left(\mathrm{rc}_{4}|| \mathrm{rc}_{3}\left\|\mathrm{rc}_{2}\right\| \mathrm{rc}_{1}\left\|\mathrm{rc}_{0}\right\| \mathrm{rc}_{5} \oplus \mathrm{rc}_{4} \oplus 1\right) .
$$

The six bits are initialized to zero, and updated before used in a given round. The bits from the LFSR are arranged into a $4 \times 4$ array and only the first column of the state is affected by the LFSR bits, i.e.,

$$
\left[\begin{array}{cccc}
c_{0} & 0 & 0 & 0 \\
c_{1} & 0 & 0 & 0 \\
c_{2} & 0 & 0 & 0 \\
0 & 0 & 0 & 0
\end{array}\right],
$$


with $c_{2}=0 \times 2$ and $\left(c_{0}, c_{1}\right)=\left(0\|0\| 0\|0\| \mathrm{rc}_{3}\left\|\mathrm{rc}_{2}\right\| \mathrm{rc}_{1}\left\|\mathrm{rc}_{0}, 0\right\| 0\|0\| 0\|0\| 0\left\|\mathrm{rc}_{5}\right\| \mathrm{rc}_{4}\right)$.

The round constants are combined with the state, respecting array positioning, using bitwise exclusive-or. The values of the $\left(\mathrm{rc}_{5}, \mathrm{rc}_{4}, \mathrm{rc}_{3}, \mathrm{rc}_{2}, \mathrm{rc}_{1}, \mathrm{rc}_{0}\right)$ constants for each round are given in Table 2 below, encoded to byte values for each round, with $\mathrm{rc}_{0}$ being the least significant bit.

Table 2: SKINNY Round Constants.

\begin{tabular}{|c|c|}
\hline Rounds & Constants \\
\hline $1-16$ & $01,03,07,0 \mathrm{~F}, 1 \mathrm{~F}, 3 \mathrm{E}, 3 \mathrm{D}, 3 \mathrm{~B}, 37,2 \mathrm{~F}, 1 \mathrm{E}, 3 \mathrm{C}, 39,33,27,0 \mathrm{E}$ \\
\hline $17-32$ & $1 \mathrm{D}, 3 \mathrm{~A}, 35,2 \mathrm{~B}, 16,2 \mathrm{C}, 18,30,21,02,05,0 \mathrm{~B}, 17,2 \mathrm{E}, 1 \mathrm{C}, 38$ \\
\hline $33-48$ & $31,23,06,0 \mathrm{D}, 1 \mathrm{~B}, 36,2 \mathrm{D}, 1 \mathrm{~A}, 34,29,12,24,08,11,22,04$ \\
\hline $49-62$ & $09,13,26,0 \mathrm{C}, 19,32,25,0 \mathrm{~A}, 15,2 \mathrm{~A}, 14,28,10,20$ \\
\hline
\end{tabular}

AddRoundTweakey. The first and second rows of all tweakey arrays are extracted and bitwise exclusive-ored to the ciphers internal state, respecting the array positioning. More formally, for $i=\{0,1\}$ and $j=\{0,1,2,3\}$, we have:

- $I S_{i, j}=I S_{i, j} \oplus T K 1_{i, j} \oplus T K 2_{i, j}$ for SKINNY-128-256,

- $I S_{i, j}=I S_{i, j} \oplus T K 1_{i, j} \oplus T K 2_{i, j} \oplus T K 3_{i, j}$ for SKINNY-128-384.

Then, the tweakey arrays are updated as follows: First, a permutation $P_{T}$ is applied on the cells positions of all tweakey arrays: for all $0 \leq i \leq 15$, we set $T K z_{i} \leftarrow T K z_{P_{T}[i]}$ with

$$
P_{T}=[9,15,8,13,10,14,12,11,0,1,2,3,4,5,6,7],
$$

for $z \in\{1,2\}$ (resp., $z \in\{1,2,3\}$ ). This corresponds to the following reordering of the matrix cells, where indices are taken row-wise:

$$
(0, \ldots, 15) \stackrel{P_{T}}{\longmapsto}(9,15,8,13,10,14,12,11,0,1,2,3,4,5,6,7) .
$$

Finally, every cell of the first and second rows of TK2 (resp., TK2 and TK3) are individually updated with an LFSR. The LFSRs used are given in Table 3 ( $x_{0}$ stands for the LSB of the cell).

Table 3: The LFSRs used in SKINNY to generate the round tweakeys. The $T K$ parameter gives the number of the corresponding tweakey word in the cipher.

\begin{tabular}{lll}
\hline TK & $s$ & \multicolumn{1}{c}{ LFSR } \\
\hline$T K 2$ & 8 & $\left(x_{7}\left\|x_{6}\right\| x_{5}\left\|x_{4}\right\| x_{3}\left\|x_{2}\right\| x_{1} \| x_{0}\right) \rightarrow\left(x_{6}\left\|x_{5}\right\| x_{4}\left\|x_{3}\right\| x_{2}\left\|x_{1}\right\| x_{0} \| x_{7} \oplus x_{5}\right)$ \\
$T K 3$ & 8 & $\left(x_{7}\left\|x_{6}\right\| x_{5}\left\|x_{4}\right\| x_{3}\left\|x_{2}\right\| x_{1} \| x_{0}\right) \rightarrow\left(x_{0} \oplus x_{6}\left\|x_{7}\right\| x_{6}\left\|x_{5}\right\| x_{4}\left\|x_{3}\right\| x_{2} \| x_{1}\right)$ \\
\hline
\end{tabular}

ShiftRows. As in the AES, in this layer, the rows of the cipher state cell array are rotated. More precisely, the second, third, and fourth cell rows are rotated by 1,2 and 3 positions to the right, respectively. In other words, a permutation $P$ is applied on the cells positions of the cipher internal state cell array: for all $0 \leq i \leq 15$, we set $I S_{i} \leftarrow I S_{P[i]}$ with

$$
P=[0,1,2,3,7,4,5,6,10,11,8,9,13,14,15,12] .
$$


MixColumns. Each column of the cipher internal state array is multiplied by the following binary matrix $\mathbf{M}$ :

$$
\mathbf{M}=\left(\begin{array}{llll}
1 & 0 & 1 & 1 \\
1 & 0 & 0 & 0 \\
0 & 1 & 1 & 0 \\
1 & 0 & 1 & 0
\end{array}\right)
$$

The final value of the internal state array provides the ciphertext with cells being unpacked in the same way as the packing during initialization. Test vectors for SKINNY-128-256 and SKINNY-128-384 are provided in Appendix B. Note that decryption is very similar to encryption as all cipher components have very simple inverses (SubCells and MixColumns are based on a generalized Feistel structure, so their respective inverse is straightforward to deduce and can be implemented with the exact same number of operations).

\subsection{The AEAD Scheme SKINNY-AEAD}

The authenticated encryption scheme adopts the OCB3 mode using either SKINNY-128-384 or SKINNY-128-256 as the underlying tweakable block cipher, depending on the member as shown in Table 1. In the following, we provide the detailed specification of the scheme. Let SKINNY-128-384 $t k(P)$ denote the encryption of a plaintext $P$ under the tweakey $t k$ with the SKINNY-128-384 algorithm and let SKINNY-128-256 $t k(P)$ be the encryption of a plaintext $P$ under the tweakey $t k$ with the SKINNY-128-256 algorithm. Let further SKINNY-128-384 ${ }_{t k}^{-1}(C)$ (resp. SKINNY-128-256 ${ }_{t k}^{-1}(C)$ ) denote the decryption of a ciphertext $C$ under the tweakey $t k$ with the SKINNY-128-384 (resp. SKINNY-128-256) algorithm.

By $(N, A, M)$, we denote the tuple of a nonce $N$, associated data $A$ and a message $M$, where $A$ and $M$ can be of arbitrary length (including empty).

\subsubsection{SKINNY-AEAD Based on SKINNY-128-384}

This case applies to our primary member (M1), as well as M2, M3, and M4 (refer to Table 1).

Domain Separation. We first define a 1-byte string that ensures independence of tweakable block cipher calls for different kinds of computations (i.e., domain separation) and also for different SKINNY-AEAD members. Let $b_{7}\left\|b_{6}\right\| b_{5}\left\|b_{4}\right\| b_{3}\left\|b_{2}\right\| b_{1} \| b_{0}$ be the bitwise representation of this byte, where $b_{7}$ is the MSB and $b_{0}$ is the LSB (see also Figure 2). Then, we use the following convention:

- $b_{7}$ to $b_{5}$ are always fixed to 0 ,

- $b_{4}$ encodes the nonce size $n_{\ell} \in\{0,1\}$, where $n_{\ell}$ is set to 0 if the nonce size is 128 bits and 1 if the nonce size is 96 bits,

- $b_{3}$ encodes the tag size $t_{\ell} \in\{0,1\}$, where $t_{\ell}$ is set to 0 if the tag size is 128 bits and 1 if the tag size is 64 bits,

- $b_{2}$ to $b_{0}$ are used for the actual domain separation, which is further specified as follows:

- 000: encryption of a full message block,

- 001: encryption of a partial message block,

- 010: computation of a full associated data block, 
- 011: computation of a partial associated data block,

- 100: generation of a tag if the message size in bits is a multiple of 128 ,

- 101: generation of a tag if the message size in bits is not a multiple of 128 .

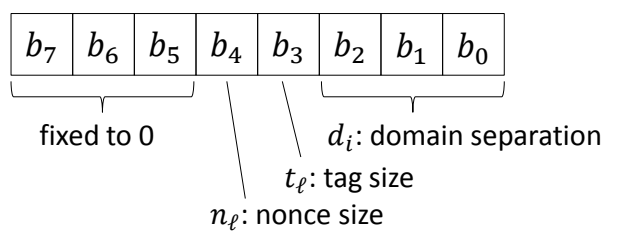

Figure 2: Domain separation and distinction of the different members.

Note that the nonce size $\left(b_{4}\right)$ and the tag size $\left(b_{3}\right)$ are fixed during the computation of a single tuple of nonce $N$, associated data $A$ and message $M$, while $b_{2}$ to $b_{0}$ vary across a computation of a single $(N, A, M)$.

In the following paragraphs, we specify the computations of a ciphertext $C$ and a tag tag for a given $(N, A, M)$, key $K, n_{\ell}$, and $t_{\ell}$. For simplicity, we denote this single byte by $d_{0}, d_{1}, d_{2}, d_{3}, d_{4}$, or $d_{5}$ depending on the 3 -bit value for the domain separation, i.e.:

$$
\begin{aligned}
& d_{0}=000 n_{\ell} t_{\ell} 000, \\
& d_{1}=000 n_{\ell} t_{\ell} 001, \\
& d_{2}=000 n_{\ell} t_{\ell} 010, \\
& d_{3}=000 n_{\ell} t_{\ell} 011, \\
& d_{4}=000 n_{\ell} t_{\ell} 100, \\
& d_{5}=000 n_{\ell} t_{\ell} 101 .
\end{aligned}
$$

Associated Data Processing. The computation for the associated data is depicted in Figure 3. If the byte-length of $A$ is not a multiple of the block size (i.e., 16 bytes), it has to be padded. In particular, if $|A|$ denotes the length of $A$ in bit, let $A=$ $A_{0}\left\|A_{1}\right\| \ldots\left\|A_{\ell_{a}-1}\right\| A_{\ell_{a}}$ with $\left|A_{i}\right|=128$ for $i \in\left\{0, \ldots, \ell_{a}-1\right\}$ and $\left|A_{\ell_{a}}\right|<128$. Note that if $|A|$ is a multiple of $128,\left|A_{\ell_{a}}\right|$ is set to the empty string $\epsilon$ and no padding is applied. Otherwise, we apply the padding pad10* to $A_{\ell_{a}}$ which is defined as

$$
\operatorname{pad} 10^{*}: X \mapsto X\|1\| 0^{127-|X| \bmod 128} \text {. }
$$

Each associated data block $A_{i}$ is processed in parallel by SKINNY-128-384 as a plaintext input under a 384-bit tweakey, where the structure of the 384-bit tweakey is as follows.

- The tweakey bytes $t k_{0}, \ldots, t k_{15}$ store 8 bytes from a 64 -bit LFSR, followed by 7 bytes of zeros, and then the single byte for the domain separation $\left(d_{2}\right.$ or $d_{3}$ whether it is a padded block). The 64-bit LFSR plays the role of a block counter. It is defined as follows: Let $x_{63}\left\|x_{62}\right\| x_{61}\|\cdots\| x_{2}\left\|x_{1}\right\| x_{0}$ denote the 64 -bit state of the LFSR. It is initialized to $\operatorname{LFSR}_{0}=0^{63} \| 1$ and updated as $\operatorname{LFSR}_{t+1}=\operatorname{upd}_{64}\left(\operatorname{LFSR}_{t}\right)$, where the update function $\operatorname{upd}_{64}$ is defined by the polynomial $x^{64}+x^{4}+x^{3}+x+1$ as

$$
\operatorname{upd}_{64}: x_{63}\left\|x_{62}\right\| \ldots\left\|x_{1}\right\| x_{0} \quad \longrightarrow \quad y_{63}\left\|y_{62}\right\| \ldots\left\|y_{1}\right\| y_{0}
$$


with:

$$
\begin{aligned}
& y_{i} \leftarrow x_{i-1} \text { for } i \in\{63,62, \ldots, 1\} \backslash\{4,3,1\}, \\
& y_{4} \leftarrow x_{3} \oplus x_{63}, \\
& y_{3} \leftarrow x_{2} \oplus x_{63}, \\
& y_{1} \leftarrow x_{0} \oplus x_{63}, \\
& y_{0} \leftarrow x_{63} .
\end{aligned}
$$

Before loaded in the tweakey state, the order of the bytes of the LFSR state is reversed, i.e., $t k_{0}\left\|t k_{1}\right\| \ldots\left\|t k_{15}=\operatorname{rev}_{64}\left(\operatorname{LFSR}_{t}\right)\right\| 0^{56} \| d_{2}$ (resp. $t k_{15}=d_{3}$ for the last padded block), where rev $_{64}$ is defined by

$$
\operatorname{rev}_{64}: x_{7}\left\|x_{6}\right\| x_{5}\left\|x_{4}\right\| x_{3}\left\|x_{2}\right\| x_{1}\left\|x_{0} \mapsto x_{0}\right\| x_{1}\left\|x_{2}\right\| x_{3}\left\|x_{4}\right\| x_{5}\left\|x_{6}\right\| x_{7} \quad\left(\forall i:\left|x_{i}\right|=8\right) \text {. }
$$

- The tweakey bytes $t k_{16}\left\|t k_{17}\right\| \ldots \| t k_{31}$ store the nonce $N$. If $n_{\ell}=1$, i.e., if the nonce size is 96 bits, 32-bit zeros are appended to $N$, thus, $t k_{16}\left\|t k_{17}\right\| \ldots\left\|t k_{31}=N\right\| 0^{32}$.

- The tweakey bytes $t k_{32}\left\|t k_{33}\right\| \ldots \| t k_{47}$ store the 128-bit key $K$.

The XOR sum of each block's output is stored as Auth, which is later used in the final authentication tag computation.

Remind that if the size of $A$ is not a multiple of 128 bits, we use the domain separation byte $d_{3}$ to process the last padded block.

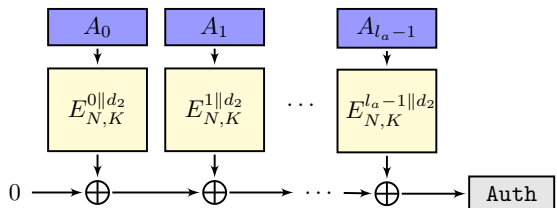

(a) Without padding

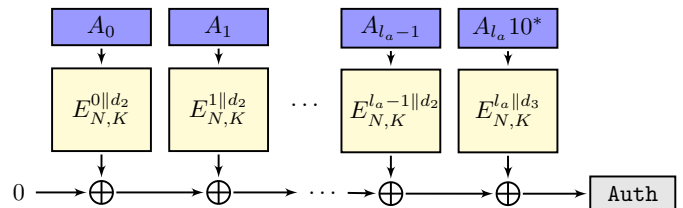

(b) With padding.

Figure 3: Handling of the associated data: in the case where the associated data is a multiple of the block size, no padding is needed. In the figures, $E$ refers to SKINNY-128-384. For simplicity, we denote the block counter by $0 \ldots, \ell_{a}-1$ (resp., $0, \ldots, \ell_{a}$ ) but actually refer to the state of the LFSRs serving as a block counter.

Encryption. The encryption of $M$ is depicted in Figure 4 and Figure 5. First, suppose that the size of $M$ in bits is a multiple of 128 (Figure 4). In that case, $M$ is parsed into 128-bit blocks $M_{0}, M_{1}, M_{2}, \ldots, M_{\ell_{m}-1}$ and no padding is applied. Each message block $M_{i}$ is processed by SKINNY-128-384 as a plaintext input under a particular 384-bit tweakey and the output is taken as the corresponding ciphertext block. The structure of the 384-bit tweakey differs from the associated data processing only by the domain separation byte. Here, the byte $t k_{15}$ is fixed to $d_{0}$ instead of $d_{2}$.

To produce the tag, the XOR sum of the plaintext blocks noted $\Sigma$ is computed and then encrypted by SKINNY-128-384, where the 384-bit tweakey is analogously defined as $t k_{0}\left\|t k_{1}\right\| \ldots\left\|t k_{47}=\operatorname{rev}_{64}\left(\operatorname{LFSR}_{\ell_{m}}\right)\right\| 0^{56}\left\|d_{4}\right\| N \| K$. Finally, the output of this encryption is XORed with Auth. If $t_{\ell}=0$, i.e., the tag size is 128 bits, the result of this XOR is a tag. If $t_{\ell}=1$, i.e., the tag size is 64 bits, the result of this XOR is truncated by trunc 64 to 64 bit, where the truncation functions trunc $_{i}$ are defined for inputs of length at least $i$ by

$$
\operatorname{trunc}_{i}: X=x_{0}\left\|x_{1}\right\| \ldots\left\|x_{|X|-1} \mapsto x_{0}\right\| x_{1}\|\ldots\| x_{i-1} .
$$




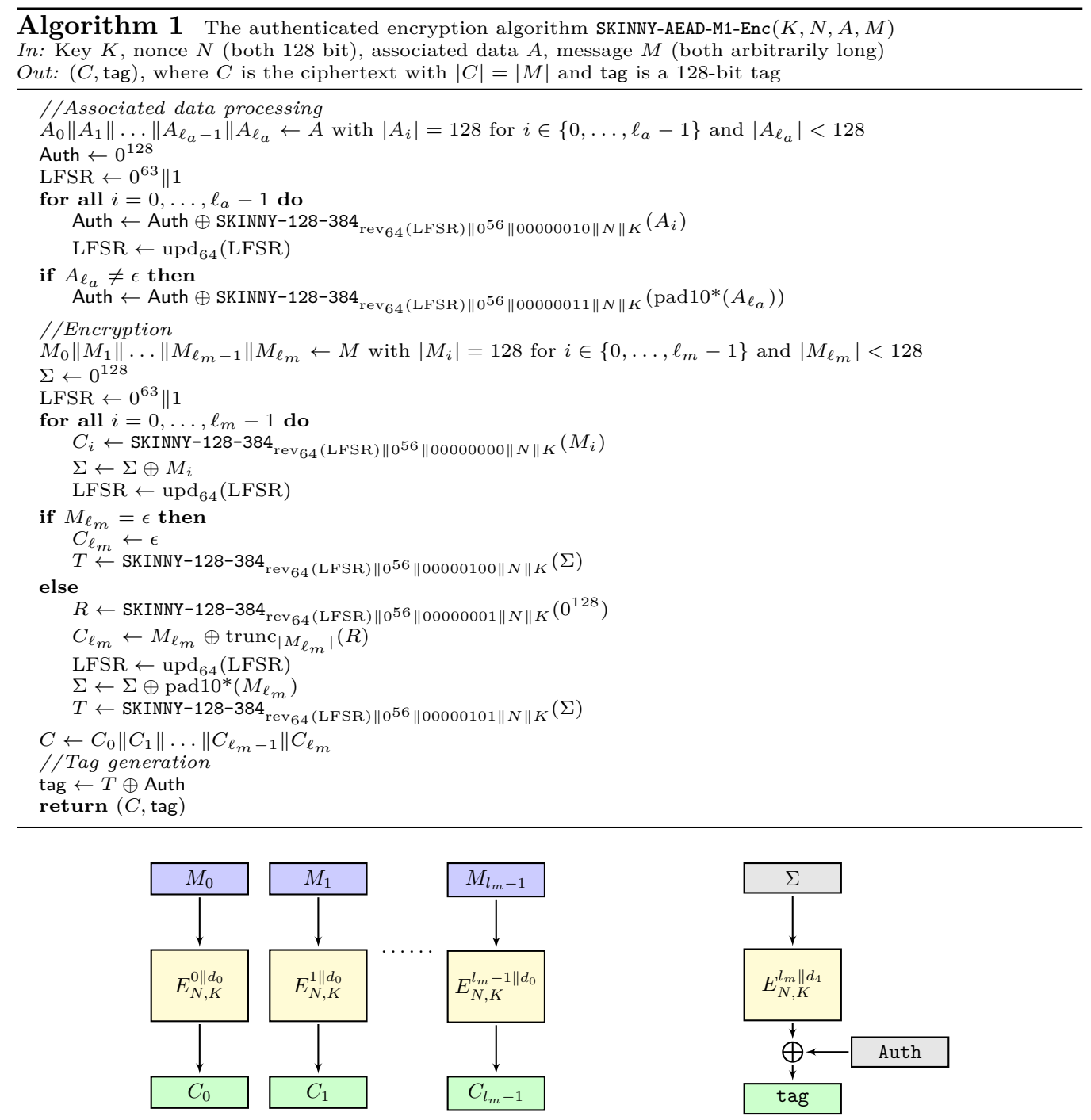

Figure 4: Encryption of SKINNY-AEAD with SKINNY-128-384 without padding when $t_{\ell}=128$. Again, $E$ refers to SKINNY-128-384. For simplicity, we denote the block counter by $0, \ldots, \ell_{m}$ but actually refer to the state of the LFSRs serving as a block counter.

In the case $|M|$ is not a multiple of 128 (Figure 5), the same padding pad10* as for the associated data is applied to $M$. In particular, $M$ is split into $M=M_{0}\left\|M_{1}\right\| \ldots\left\|M_{\ell_{m}-1}\right\| M_{\ell_{m}}$ with $\left|M_{i}\right|=128$ for $i \in\left\{0, \ldots, \ell_{m}-1\right\}$ and $0<\left|M_{\ell_{m}}\right|<127$. The processing of the message blocks $M_{i}, i \in\left\{0, \ldots, \ell_{m}-1\right\}$ is the same as in the case described above. The last ciphertext block $C_{\ell_{m}}$ is computed as the XOR sum of the encryption of 0 with SKINNY-128-384 under the 384-bit tweakey $t k_{0}\left\|t k_{1}\right\| \ldots\left\|t k_{47}=\operatorname{rev}_{64}\left(\operatorname{LFSR}_{\ell_{m}}\right)\right\| 0^{56}\left\|d_{1}\right\| N \| K$ (truncated to $\left|M_{\ell_{m}}\right|$ bits) with the plaintext block $M_{\ell_{m}}$.

For the tag computation, the checksum is computed as $M_{0} \oplus M_{1} \oplus \cdots \oplus M_{\ell_{m}-1} \oplus$ $\operatorname{pad10}\left(M_{\ell_{m}}\right)$ and it is encrypted with SKINNY-128-384 under the 384-bit tweakey

$$
\operatorname{rev}_{64}\left(\operatorname{LFSR}_{\ell_{m+1}}\right)\left\|0^{56}\right\| d_{5}\|N\| K \text {. }
$$

Similar as for the unpadded case, the encryption is XORed with Auth and truncated in the same way as described above if $t_{\ell}=1$. 


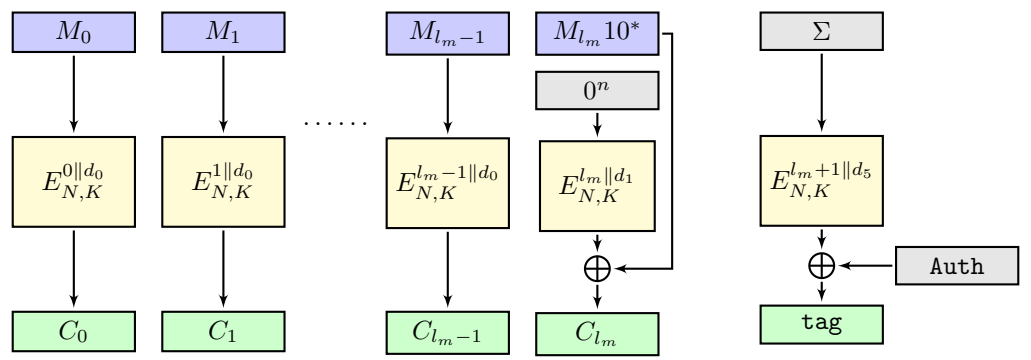

Figure 5: Encryption of SKINNY-AEAD with SKINNY-128-384 with padded message when $t_{\ell}=128$. The last ciphertext block $C_{l_{m}}$ is further truncated to have the same size as $M_{l_{m}}$. Again, we denote the block counter by $0, \ldots, \ell_{m}+1$ but actually refer to the state of the LFSRs serving as a block counter.

Decryption. The decryption and tag verification procedure for given $(K, N, A, C$, tag) is straightforward.

Formally, we provide the algorithms of the authenticated encryption members M1, M2, M3, and M4, together with their decryption and tag verification procedure, in Algorithms 1, 2 (in the main text), 5, 6, 7, 8 and 9, 10 (in Appendix C), respectively.

\subsubsection{SKINNY-AEAD with SKINNY-128-256}

This case applies to the members M5 and M6 (refer to Table 1). It is very similar to the previous case, the main difference being the definition of the tweakey states due to their smaller sizes.

Domain Separation. The domain separation is exactly the same as in the previous case. Note that $b_{4}$ is always fixed to 1 as only 96-bit nonces can be used in the members M5 and M6.

Associated Data Processing. The computation for associated data $A$ is very similar to the previous case. The difference is that each associated data block $A_{i}$ is processed by SKINNY-128-256 as a plaintext input under a 256-bit tweakey, where the structure of the 256-bit tweakey is as follows.

- The tweakey bytes $t k_{0}, \ldots, t k_{15}$ store 3 bytes from a 24 -bit LFSR, the single byte for the domain separation, followed by the 12-byte nonce $N$. The byte for the domain separation is fixed to $d_{2}$, i.e., $0001 t_{\ell} 010$, for a non-padded block and to $d_{3}=0001 t_{\ell} 011$ for a padded block. The 24-bit LFSR is defined below.

Let $x_{23}\left\|x_{22}\right\| x_{21}\|\cdots\| x_{2}\left\|x_{1}\right\| x_{0}$ denote the 24 bits of the LFSR. It is initialized to $L F S R_{0}=0^{23} 1$ and updated as $\operatorname{LFSR}_{t+1}=\operatorname{upd}_{24}\left(\mathrm{LFSR}_{t}\right)$, where the update function $\operatorname{upd}_{24}$ is defined by the polynomial $x^{24}+x^{4}+x^{3}+x+1$ as

$$
\operatorname{upd}_{24}: x_{23}\left\|x_{22}\right\| \ldots\left\|x_{1}\right\| x_{0} \mapsto y_{23}\left\|y_{22}\right\| \ldots\left\|y_{1}\right\| y_{0}
$$

with

$$
\begin{aligned}
& y_{i} \leftarrow x_{i-1} \text { for } i \in\{23,22, \ldots, 1\} \backslash\{4,3,1\}, \\
& y_{4} \leftarrow x_{3} \oplus x_{23}, \\
& y_{3} \leftarrow x_{2} \oplus x_{23}, \\
& y_{1} \leftarrow x_{0} \oplus x_{23}, \\
& y_{0} \leftarrow x_{23} .
\end{aligned}
$$




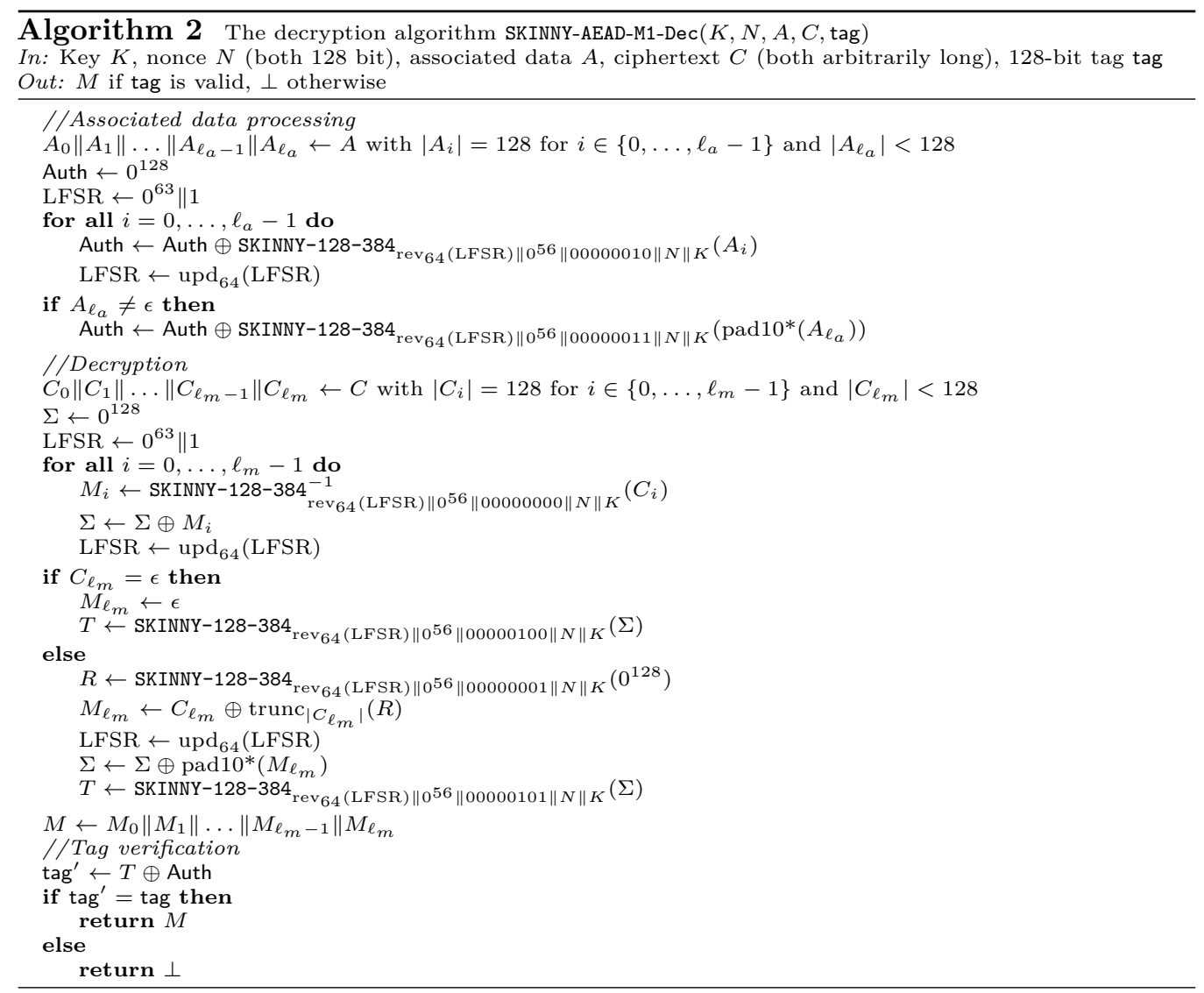

Before loaded in the tweakey state, the order of the bytes of the LFSR state is reversed, i.e., $t k_{0}\left\|t k_{1}\right\| \ldots\left\|t k_{15}=\operatorname{rev}_{24}\left(\mathrm{LFSR}_{t}\right)\right\| d_{2} \| N$ (resp. $t k_{15}=d_{3}$ for the last padded block), where $\operatorname{rev}_{24}$ is defined by

$$
\operatorname{rev}_{24}: x_{2}\left\|x_{1}\right\| x_{0} \mapsto x_{0}\left\|x_{1}\right\| x_{2} \quad\left(\forall i:\left|x_{i}\right|=8\right)
$$

- The tweakey bytes $t k_{16}\left\|t k_{17}\right\| \ldots \| t k_{31}$ store the 128 -bit key $K$.

Encryption and Decryption. The encryption of $M$ is also very similar to the previous case. Also, decryption and tag verification is straightforward.

Formally, in Appendix C, we provide the algorithms of the authenticated encryption members M5 and M6, together with their decryption and tag verification procedure, in Algorithms 11, 12 and 13, 14, respectively.

\subsubsection{Remarks for Further Extension}

Here, we explain two additional features of our AEAD schemes, which are not officially included in the NIST submission but can be implemented efficiently depending on the user's demand.

Supporting More than $\mathbf{2}^{\mathbf{6 4}}$ blocks with SKINNY-128-384. Recall that in the members based on SKINNY-128-384, the tweakey bytes $t k_{0}, \ldots, t k_{15}$ store 8 bytes for a 64 -bit LFSR, followed by 7 bytes of zeros, and then a single byte for the domain separation. If the user wants to support input data of more than $2^{64}$ blocks, it is possible to replace the 7 
bytes of zeros by the following 56-bit LFSR. Note that this LFSR would be updated every $2^{64}$ blocks, hence very rarely in comparison to the 64 -bit LFSR. Let $x_{55}\left\|x_{54}\right\| \cdots\left\|x_{1}\right\| x_{0}$ denote the 56 bits of the 56-bit LFSR. It is initialized to $\operatorname{LFSR}_{0}=0^{55} \| 1$ and updated as $\operatorname{LFSR}_{t+1}=\operatorname{upd}_{56}\left(\operatorname{LFSR}_{t}\right)$, where the update function upd ${ }_{56}$ is defined by the polynomial $x^{56}+x^{7}+x^{4}+x^{2}+1$ as

$$
\operatorname{upd}_{56}: x_{55}\left\|x_{54}\right\| \ldots\left\|x_{1}\right\| x_{0} \quad \longrightarrow \quad y_{55}\left\|y_{54}\right\| \ldots\left\|y_{1}\right\| y_{0}
$$

with:

$$
\begin{aligned}
y_{i} & \leftarrow x_{i-1} \text { for } i \in\{55,54, \ldots, 1\} \backslash\{7,4,2\}, \\
y_{7} & \leftarrow x_{6} \oplus x_{55}, \\
y_{4} & \leftarrow x_{3} \oplus x_{55}, \\
y_{2} & \leftarrow x_{1} \oplus x_{55}, \\
y_{0} & \leftarrow x_{55} .
\end{aligned}
$$

We stress that this additional functionality is only available in SKINNY-128-384-based members, and cannot be adopted in SKINNY-128-256-based members.

Acceleration of Associated Data Processing. When associated data $A$ is processed, we fix 128 bits or 96 bits of the tweakey state to the nonce value $N$ for SKINNY-128-384- and SKINNY-128-256-based members, respectively. We note that it is not strictly necessary to include $N$ during the associated data processing, hence a potential acceleration of the associated data processing could replace $N$ with bits from $A$. This would reduce the number of tweakable block cipher calls for processing $A$. In particular, the number of calls could be halved in SKINNY-128-384-based members.

\subsection{The Hash Functionality SKINNY-Hash}

Overall, the SKINNY-Hash family consists of the function-based sponge constructions SKINNY-tk3-Hash and SKINNY-tk2-Hash, in which underlying functions are built with SKINNY-128-384 and SKINNY-128-256, respectively. We recall here that the sponge construction [BDPA11] can be based on a cryptographic function as well as a cryptographic permutation.

\subsection{1 $F_{384}$ : 384-bit to 384-bit function}

We build a function $F_{384}:\{0,1\}^{384} \rightarrow\{0,1\}^{384}$ based on SKINNY-128-384. Let $x \in$ $\{0,1\}^{384}$ be an input to $F_{384}$. Let SKINNY-128-384tk $(P)$ be the encryption of a plaintext $P$ under a tweakey $t k$ with the SKINNY-128-384 algorithm. The output of $F_{384}$ is computed as follows (see also Figure 6):

$$
F_{384}(x)=\operatorname{SKINNY}-128-384_{x}\left(0^{128}\right)\left\|\operatorname{SKINNY}-128-384_{x}\left(0^{7}\|1\| 0^{120}\right)\right\| \text { SKINNY-128-384 }{ }_{x}\left(0^{6}\|1\| 0^{121}\right) .
$$

\subsection{2 $F_{256}: 256-$ bit to 256 -bit function}

We build a function $F_{256}:\{0,1\}^{256} \rightarrow\{0,1\}^{256}$ based on SKINNY-128-256. For this, let SKINNY-128-256 ${ }_{t k}(P)$ be the encryption of a plaintext $P$ under a tweakey $t k$ with the SKINNY-128-256 algorithm. The output of $F_{256}$ is computed as follows (see also Figure 6):

$$
F_{256}(x)=\operatorname{SKINNY}-128-256_{x}\left(0^{128}\right) \| \operatorname{SKINNY}-128-256_{x}\left(0^{7}\|1\| 0^{120}\right) .
$$




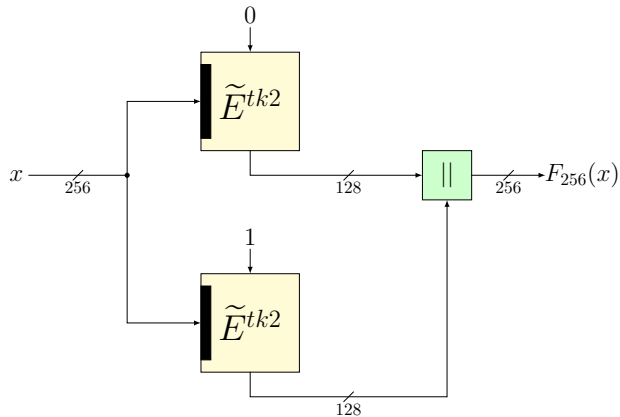

(a) Construction of $F_{256}$.

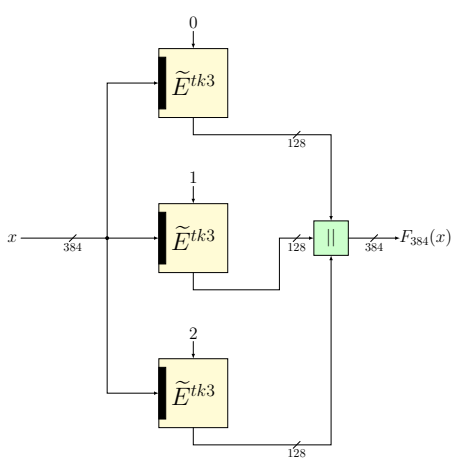

(b) Construction of $F_{384}$.

Figure 6: Construction of the functions $F_{256}$ and $F_{384}$ used in SKINNY-tk2-Hash and SKINNY-tk3-Hash, respectively.

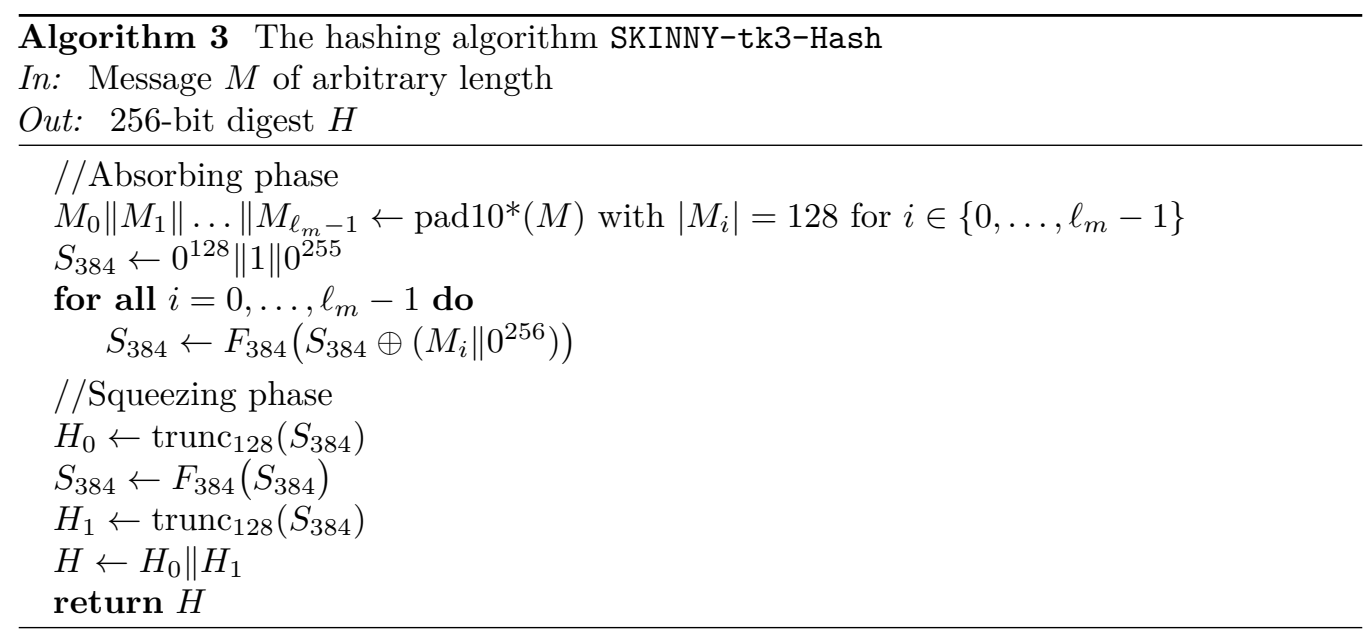

\subsubsection{SKINNY-tk3-Hash}

The computation of SKINNY-tk3-Hash simply follows the well-known sponge construction. Differently from many of existing instantiations, we use the function $F_{384}$ as an underlying primitive. The construction is illustrated in Figure 7.

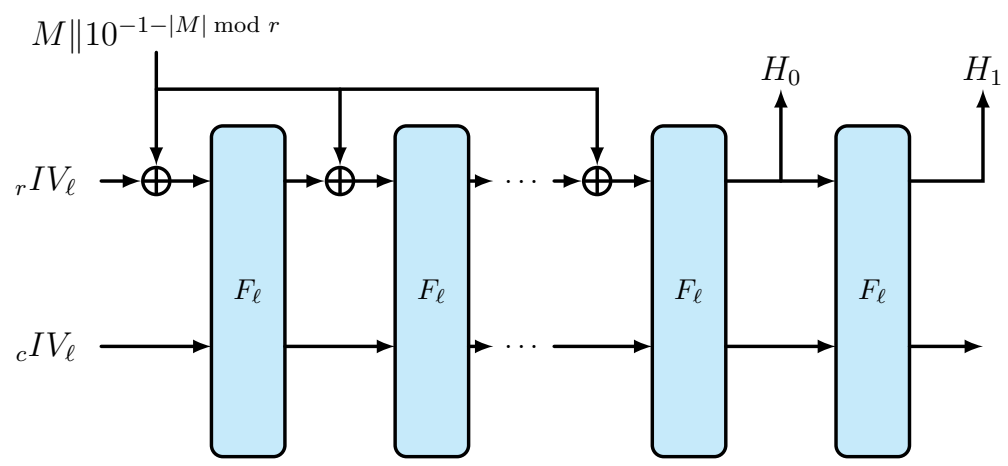

Figure 7: The structure of SKINNY-Hash based on a sponge. 
The 384-bit state, $S_{384}$, is divided into 128-bit rate and 256-bit capacity, which are initialized to the following values:

$$
\begin{aligned}
{ }_{r} I V_{384} & =0^{128}, \\
{ }_{c} I V_{384} & =10^{255}, \\
S_{384} & ={ }_{r} I V_{384} \|_{c} I V_{384} .
\end{aligned}
$$

The padding pad10* is applied to an input message $M$ (note that the padding is always applied, even if $|M|$ is already a multiple of 128). The message blocks $M_{i}$ are XORed to the outer part of the state during the absorbing phase.

After the absorbing phase, the 128 bits of the rate are extracted as the first 128 bits of the 256-bit digest. Then, $S_{384} \leftarrow F_{384}\left(S_{384}\right)$ is applied once again and the 128 bits of the rate are extracted as the last 128 bits of the 256 -bit digest. The formal algorithm is specified in Algorithm 3.

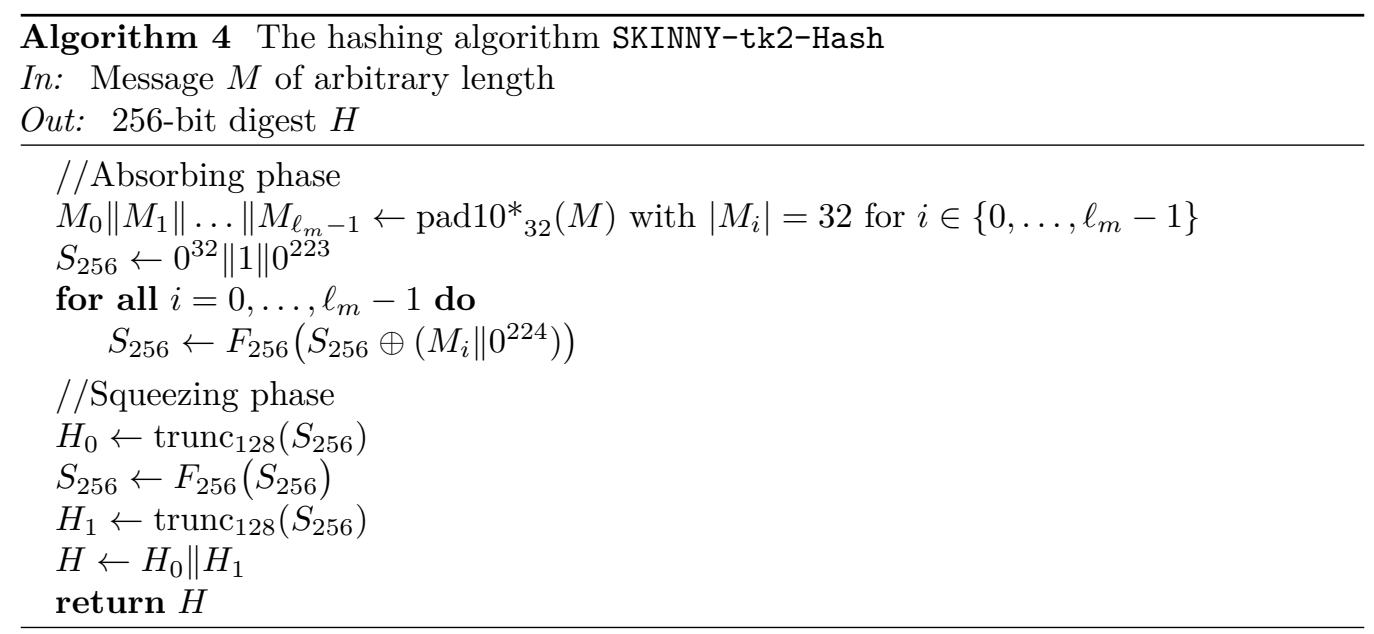

\subsubsection{SKINNY-tk2-Hash}

The 256-bit state $S_{256}$, is divided into a 32 -bit outer part and a 224-bit inner part, which are initialized to the following values:

$$
\begin{aligned}
{ }_{r} I V_{256} & =0^{32}, \\
{ }_{c} I V_{256} & =10^{223}, \\
S_{256} & ={ }_{r} I V_{256} \|_{c} I V_{256} .
\end{aligned}
$$

A difference with the previous case is that the message $M$ now has to be padded such that its length is a multiple of 32 bits. Therefore, we apply the padding function pad10* ${ }_{32}$ which is defined as

$$
\text { pad10* }{ }_{32}: X \mapsto X\|1\| 0^{31-|X| \bmod 32} .
$$

The message blocks $M_{i}$ are XORed to the outer part of the state during the absorbing phase. After the absorbing phase, the first 128 bits of the state are extracted as the first 128 bits of the 256-bit digest. Then, $S_{256} \leftarrow F_{256}\left(S_{256}\right)$ is applied once again and the first 128 bits of the state are extracted as the last 128 bits of the 256 -bit digest. This means that in the squeezing phase, the rate is extended to 128 bits and the capacity is reduced to 128 bits. The formal algorithm is specified in Algorithm 4. 


\subsubsection{Table of Parameters and Security of SKINNY-Hash}

For a summary, parameters of SKINNY-Hash are listed in Table 4.

Table 4: Parameters for SKINNY-Hash. The number of blocks of the first preimage is denoted by $L$.

\begin{tabular}{|c|c|c|c|c|c|c|c|}
\hline \multirow[t]{2}{*}{ Algorithm } & \multirow[t]{2}{*}{ State size } & \multicolumn{2}{|c|}{ Absorb } & \multicolumn{2}{|c|}{ Squeeze } & \multirow{2}{*}{$\begin{array}{l}\text { Security } \\
\text { (collision) }\end{array}$} & \multirow{2}{*}{$\begin{array}{c}\text { Security } \\
\text { (2nd preimage) }\end{array}$} \\
\hline & & Rate & Capacity & Rate & Capacity & & \\
\hline SKINNY-tk3-Hash & 384 & 128 & 256 & 128 & 256 & 128 & $256-\log _{2}(L)$ \\
\hline SKINNY-tk2-Hash & 256 & 32 & 224 & 128 & 128 & 112 & $224-\log _{2}(L)$ \\
\hline
\end{tabular}

\section{Security Claims}

We provide our security claims for the different variants of SKINNY-AEAD and SKINNY-Hash in Table 5. Basically, for all versions of SKINNY-AEAD, we claim full 128-bit security for key recovery, confidentiality and integrity (unless the tag size is smaller than 128 bits, in which case the integrity security claims drop to the tag size) in the nonce-respecting model. For all versions of SKINNY-Hash, we claim that it is hard to find a collision, preimage or second-preimage with substantially less than $2^{c / 2}$ hash evaluations, where $c$ represents the capacity bitsize ( $c=256$ for M5 and $c=224$ for M6).

One can see that we do claim full 128-bit security for all variants of SKINNY-AEAD with a tag size of 128 bit for a nonce-respecting user. More precisely, confidentiality is perfectly guaranteed and the forgery probability is $2^{-\tau}$, where $\tau$ denotes the tag size, independently of the number of blocks of data in encryption queries made by the adversary. This is very different than other modes like AES-GCM [MV04] or OCB3 [KR11], which only ensure birthday-bound security. In comparison, OCB3 only provides security up to the birthday bound, more precisely up to roughly $2^{n / 2}$ blocks of data since it relies on XE/XEX (a construction of a tweakable block cipher from a standard block cipher with security only up to the birthday bound). To give a numerical example, with $2^{40}$ blocks ciphered (about 16 TeraBytes), one gets an advantage of about $2^{-48}$ to generate a valid tag for most operating modes in the nonce-respecting scenario. For the same amount of data, the advantage remains $2^{-128}$ for members M1/M2/M5 of SKINNY-AEAD.

Table 5: Security claims of SKINNY-AEAD and SKINNY-Hash. The bit security of our designs is expressed in terms of calls to the internal primitive, up to a small logarithmic factor.

\begin{tabular}{|c|c|c|c|c|c|c|}
\hline \multirow[b]{2}{*}{ SKINNY-AEAD (nonce-respecting) } & \multicolumn{6}{|c|}{ Security (bits) } \\
\hline & M1 & M2 & M3 & M4 & M5 & M6 \\
\hline Key recovery & 128 & 128 & 128 & 128 & 128 & 128 \\
\hline Confidentiality for the plaintext ${ }^{2}$ & 128 & 128 & 128 & 128 & 128 & 128 \\
\hline \multirow[t]{2}{*}{ Integrity for the plaintext/AD/nonce } & 128 & 128 & 64 & 64 & 128 & 64 \\
\hline & \multicolumn{6}{|c|}{ Security (bits) } \\
\hline SKINNY-Hash & \multicolumn{3}{|c|}{ M1/M2/M3/M4 } & \multicolumn{3}{|c|}{ M5/M6 } \\
\hline Collision & \multicolumn{3}{|c|}{128} & \multicolumn{3}{|c|}{112} \\
\hline (2nd)-preimage & \multicolumn{3}{|c|}{128} & \multicolumn{3}{|c|}{112} \\
\hline
\end{tabular}

\footnotetext{
${ }^{2}$ The confidentiality bit security drops to the tag length if an adversary has the decryption oracle but
} 
We assume that the total size of the associated data and the total size of the message in SKINNY-AEAD do not exceed $2^{68}$ bytes for M1/M2/M3/M4 and $2^{28}$ bytes for M5/M6. Moreover, the maximum number of messages that can be handled for a same key is $2^{n_{l}}$ for all variants of SKINNY-AEAD $\left(n_{l}=128\right.$ for M1/M3, $n_{l}=96$ for M2/M4/M5/M6). This will ensure that as long as different fixed-length nonces are used, the tweak inputs of all the tweakable block cipher calls are all unique.

Related-Cipher Attacks. By encoding the length of the tag and nonce into the domain separation, we obtain a proper separation between the SKINNY-AEAD members that employ the same instance of the SKINNY tweakable block cipher. We do not claim security against related-cipher attacks between members that employ the two different instances SKINNY-128-384 and SKINNY-128-256, e.g., M2 and M5.

Nonce-Misuse Setting. The above security claims are void under reuse of nonces. As pointed out in [VV17] for the case of Deoxys-I, the scheme is vulnerable to a universal forgery attack and a CCA decryption attack with complexity of only three queries. Because we are basically using the same mode, the attacks would apply to SKINNY-AEAD as well.

\section{Design Rationale}

For a detailed design rationale of the tweakable block ciphers SKINNY-128-256 and SKINNY-128-384, we refer to the original design paper [BJK $\left.{ }^{+} 16 \mathrm{a}, \mathrm{BJK}^{+} 16 \mathrm{~b}\right]$. We decided not to modify the primitives from their original specification. The rationale for this is that none of the extensive third-party cryptanalysis, that we discuss in detail in Section 5, pointed to any weakness of the ciphers nor any bad design choices. Indeed, all the third-party cryptanalysis confirmed the validity of the original design and its rationale. We furthermore do not see any change in the specification that would improve the ciphers to the extent that would justify such a modification. All design choices of SKINNY are optimized for its goal: Obtaining a cipher well suited for many lightweight applications.

\subsection{Rationale for the AEAD scheme}

The reason for choosing the $\Theta$ CB3 mode for the tweakable block cipher SKINNY-128-384 or SKINNY-128-256 is its provable security providing full security in the nonce-respecting setting. More precisely, for $\Theta$ CB3 using an ideal tweakable block cipher, confidentiality is perfectly guaranteed and the forgery probability is independent of the number of blocks of data in encryption/decryption queries made by the adversary. Those strong security guarantees along with its performance features are the design rationale for our choice.

We state the security bound of $\Theta C B 3$ in the nonce-respecting setting:

Lemma 2 of [KR11]. Let $\prod=\Theta C B 3[\tilde{E}, \tau]$ where $\tilde{E}$ is an ideal tweakable block cipher. Let $\mathcal{A}$ be an adversary. Then $\boldsymbol{A d v} \prod^{\text {priv }}(\mathcal{A})=0$ and $\boldsymbol{A d v} \boldsymbol{\prod}^{\text {auth }}(\mathcal{A}) \leq\left(2^{n-\tau}\right) /\left(2^{n}-1\right)$.

We denote by $\operatorname{Adv}_{\text {SKINNY-TK2 }}^{ \pm \text {prp }}(\mathcal{A})$ and $\operatorname{Adv}_{\text {SKINNY-TK3 }}^{ \pm \text {prp }}(\mathcal{A})$ the $\operatorname{SPRP}$-advantage against SKINNY-128-256 and SKINNY-128-384 respectively. Replacing the ideal tweakable block cipher with SKINNY, we have the security bounds for our members as shown in Table 6 .

On OCB and Tweakable Block Ciphers. The OCB mode was first published in [RBBK01] (i.e., OCB1). It has later been refined to OCB2 in [Rog04] and finally to OCB3 in [KR11]. That last paper describes the actual $\Theta C B 3$ framework we employ in SKINNY-AEAD by using

will not use it to decrypt any authentic ciphertext and tag directly. 
Table 6: Provable security bounds for our provided AEAD members.

Members

\begin{tabular}{|c|c|}
\hline M1, M2 & $\begin{array}{c}\operatorname{Adv}_{\prod}^{\operatorname{priv}}(\mathcal{A}) \leq \mathbf{A d} \mathbf{d v}_{\mathrm{SKINNY-TK3}}^{ \pm \operatorname{prp}}(\mathcal{A}) \\
\operatorname{Adv}_{\prod}^{\text {auth }}(\mathcal{A}) \leq\left(2^{128}-1\right)^{-1}+\operatorname{Adv}_{\mathrm{SKINNY-TK3}}^{ \pm \operatorname{prp}}(\mathcal{A})\end{array}$ \\
\hline M3, M4 & $\begin{array}{c}\mathbf{A d v}_{\prod}^{\text {priv }}(\mathcal{A}) \leq \mathbf{A d} \mathbf{d v}_{\text {SKINNY-TK3 }}^{ \pm \operatorname{prp}}(\mathcal{A}) \\
\operatorname{Adv}_{\prod}^{\operatorname{auth}}(\mathcal{A}) \leq 2^{64}\left(2^{128}-1\right)^{-1}+\mathbf{A d v}_{\text {SKINNY-TK3 }}^{ \pm \operatorname{prp}}(\mathcal{A})\end{array}$ \\
\hline M5 & $\begin{array}{c}\operatorname{Adv}_{\prod}^{\text {priv }}(\mathcal{A}) \leq \mathbf{A d} \mathbf{v}_{\text {SKINNY-TK2 }}^{ \pm \operatorname{prp}}(\mathcal{A}) \\
\operatorname{Adv}_{\prod}^{\text {auth }}(\mathcal{A}) \leq\left(2^{128}-1\right)^{-1}+\operatorname{Adv}_{\text {SKINNY-TK2 }}^{ \pm \operatorname{prp}}(\mathcal{A})\end{array}$ \\
\hline M6 & $\begin{array}{c}\operatorname{Adv}_{\prod}^{\text {priv }}(\mathcal{A}) \leq \mathbf{A d} \mathbf{v}_{\text {SKINNY-TK2 }}^{ \pm \operatorname{prp}}(\mathcal{A}) \\
\operatorname{Adv}_{\prod}^{\text {auth }}(\mathcal{A}) \leq 2^{64}\left(2^{128}-1\right)^{-1}+\mathbf{A d v}_{\text {SKINNY-TK2 }}^{ \pm \operatorname{prp}}(\mathcal{A})\end{array}$ \\
\hline
\end{tabular}

a dedicated tweakable block cipher. The classical OCB (1-3) mode does not employ a dedicated tweakable block cipher, but rather a usual block cipher in an XEX-like construction. Recently, OCB3 employed with the AES was selected as one of the winners of the CAESAR competition in the category for high-performance applications [KR16]. However, this scheme only offers birthday-bound security.

More generally, a tweakable block cipher can be described as a family of block ciphers parameterized by a public parameter, the tweak. The idea of a block cipher that gets a public parameter for achieving variability goes back to the design of the Hasty Pudding Cipher [Sch98], a submission to the AES competition. This was later formalized in the notion of a tweakable block cipher by Liskov, Rivest and Wagner at CRYPTO 2002 [LRW02]. The motivation is that independent block cipher calls are needed at the mode-of-operation level, as in OCB. Liskov, Rivest and Wagner suggested that the source of variability should be directly incorporated in the primitive itself instead at the mode-of-operation level. This is a big difference to the classical OCB mode. There, a block cipher $E$ is employed in a construction that can be understood as a tweakable block cipher (i.e., the tweakable block cipher $E_{K}^{T}$ is just defined as $\left.\left.E_{K}^{\left(T_{1}, T_{2}\right)}(x)=E_{K}\left(x \oplus T_{1}\right) \oplus T_{2}\right)\right)$. In that sense, OCB can be seen as an instance of the more general TAE mode, the tweakable authenticated encryption mode defined in [LRW02]. Indeed, Liskov, Rivest and Wagner have already proven a similar statement as Lemma 2 in [KR11]:

Theorem 3 of [LRW02]. If $\widetilde{E}$ is a secure tweakable block cipher, then $\widetilde{E}$ used in TAE mode will be unforgeable and pseudorandom.

In other words: The advantage of the adversary only comes from the distinguishing advantage of the tweakable block cipher and not from the mode.

However, the XEX construction used in OCB and also in $\Theta C B 3$ does not lead to an ideal tweakable block cipher. In fact, it only offers security up to the birthday bound. The TWEAKEY framework [JNP14] was introduced at ASIACRYPT 2014 as a method to build tweakable block ciphers from scratch (i.e., without employing an already existing underlying block cipher in a specific construction) with strong security arguments against 
differential and linear attacks. The intention of the TWEAKEY framework was to obtain beyond birthday-bound secure tweakable block ciphers and to consider key and tweak as the similar type of input (called the tweakey) such that the separation into key and tweak can be done by the user in a flexible way.

It is natural to employ a beyond-birthday secure tweakable block cipher in a mode following the TAE (resp., ЄCB3) framework in order to exploit its full strength. The thirdround CAESAR candidate Deoxys-I [JNPS16] is an already existing example following this design principle.

Our Modifications. In comparison to other modes of operation, we have decided to replace the usual block counter by an LFSR, which can be implemented with just a few operations. There is indeed no reason to use the increment function $x \mapsto x+1$ over the integers, as the security simply relies on the function having a maximal cycle. The same argument has been made for instance in the original OCB mode where Gray codes have been suggested to derive inner tweak values. Here in our AEAD mode, we adopted LFSRs with maximal periods and which can be easily implemented in both hardware and software as block counters.

\subsection{Rationale for the Hash Function Scheme}

We use the well-known sponge construction, originally presented in [BDPVA07], that is also adopted in the NIST standard SHA-3 [Dwo15] so that SKINNY-Hash can inherit its elegant features. Here, we give some arguments for our design choices with respect to the following points:

1. the sponge construction using a cryptographic function as a building block,

2. the sizes of rate and capacity, and

3. our constructions of the 256- and 384-bit functions.

Function-Based Sponge. Although a lot of existing designs following the sponge framework use a cryptographic permutation as an underlying primitive, the designers do not restrict the underlying primitive to be a permutation and show a lot of analysis for the case that the underlying primitive is a function (see [BDPA11] for a detailed documentation on cryptographic sponges and several of its variants). There does not exist any significant disadvantage to base an entire construction on a function instead of a permutation. For example, the bounds for the indifferentiability and the collision resistance are almost identical between those two constructions.

In some case, the function-based sponge constructions is more difficult to attack than the permutation based sponge constructions, because the adversary does not have access to the inverse oracle for the function based constructions. This makes a significant difference of the security against second-preimage attacks. For permutation-based constructions, second preimages can be found by generating collisions on the inner part between queries to $f$ and $f^{-1}$, which allows a generic attack with a cost of $2^{c / 2}$. For function-based designs on the other hand, the best strategy is performing a similar second-preimage attack against Merkle-Damgård constructions [KS05] that requires $\left(2^{c}\right) / L$ where $L$ is the number of blocks included in the first preimage.

Choices of Rate and Capacity. We adopt the most natural choice for SKINNY-tk3-Hash. The 256-bit capacity ensures 128-bit indifferentiability. Hence, no particular attack can be performed under $2^{128}$ computational cost.

The choice for SKINNY-tk2-Hash is very optimized for lightweight use-cases. The 224-bit capacity in the absorption phase ensures the minimum requirement of 112-bit 
security. We change the rate and capacity for the squeezing phase to reduce the number of function calls in the squeezing phase. The security in this situation is analyzed in [NO14]. Let $c$ and $c^{\prime}$ be the capacity in the absorption and the squeezing phases, respectively. It was shown that $c^{\prime}$ can be enlarged with preserving $O\left(2^{c / 2}\right)$ security for indifferentiability as long as $c^{\prime} \geq c / 2+\log _{2} c$. We are aiming at 112-bit security, hence the suitable size for $c^{\prime}$ is $c^{\prime} \geq 224 / 2+\log _{2} 224 \approx 119.8$. Because we cannot produce 256 -bit hash digest in a single block, we set $c^{\prime}=128$ so that the 256 -bit hash digest can be produced with two blocks.

The results in [NO14] are for permutation-based schemes, however we got confirmation from the authors that almost the same bound can be obtained for the function-based schemes. Strictly speaking, the bound is slightly better for the function-based schemes because the adversary cannot access the inverse oracle.

Rationale of $\boldsymbol{F}_{\mathbf{2 5 6}}$ and $\boldsymbol{F}_{\mathbf{3 8 4}}$. The security argument of the sponge construction assumes the usage of a random permutation, resp., function. To provide a secure instance of the sponge, we are going to use a function indifferentiable from random. The function $F_{256}$ is indifferentiable from a 256-bit random function up to $O\left(2^{128}\right)$ queries. Very intuitively, the only way to differentiate $F_{256}$ from an ideal object is to find the case that two simulators of $\tilde{E}^{t k 2}$ in the ideal world, one is for the plaintext 0 and the other is for the plaintext 1 , return the same output value under the same tweakey input. This occurs with probability $2^{-128}$.

The same intuitive argument applies to $F_{384}$. However, the bound is worse than the one for $F_{256}$ by a factor of 3 because the adversary now has three ways to indifferentiate the real and ideal worlds: collision of the simulators output between the first and the second simulators, between the first and third simulators, and between the second and the third simulators.

\section{Security Analysis of the SKINNY TBC}

We claim security of the SKINNY family in the related-tweakey model. We now provide an analysis of its security and then mention the best cryptanalytic results published to date.

\subsection{Differential/Linear Cryptanalysis}

In order to argue for the resistance of SKINNY against differential and linear attacks, in $\left[\mathrm{BJK}^{+} 16 \mathrm{a}\right]$ we computed lower bounds on the minimum number of active Sboxes, both in the single-key and related-tweakey models. We recall that, in a differential (resp. linear) characteristic, an Sbox is called active if it contains a non-zero input difference (resp. input mask). In contrast to the single-key model, an attacker is allowed to introduce differences (resp. masks) within the tweakey state in the related-tweakey model. We considered the three cases of choosing input differences in TK1 only, both TK1 and TK2, and in all of the tweakey states TK1, TK2 and TK3, respectively. Table 7 presents lower bounds on the number of active Sboxes for up to 30 rounds. For computing the bounds, a MixedInteger Linear Programming (MILP) model following the approach in [MWGP11, $\left.\mathrm{SHS}^{+} 13\right]$ was used.

For lower bounding the number of linear active Sboxes we used the same approach by considering the inverse of the transposed linear transformation, i.e., $\mathbf{M}^{\top}$. We only considered the single-key model as there is no cancellation of active Sboxes in linear characteristics, see [KLW17]. Note that those bounds are for single characteristic only and do not quantify any potential clustering into differentials (resp. linear hulls). 
Table 7: The bounds on the number of active Sboxes in SKINNY from $\left[\mathrm{BJK}^{+} 16 \mathrm{a}\right]$. Note that the bounds on the number of linear active Sboxes in the single-key model are also valid in the related-tweakey model. In case the MILP optimization was too long, upper bounds are given between parentheses. The bounds indicated by $\star$ were obtained from $\left[\mathrm{ABI}^{+} 18\right]$ in which the authors used Matsui's algorithm for obtaining the minimum number of active Sboxes. The bounds indicated by $\dagger$ were obtained from [NSS20] by using MILP with more informative constraints.

\begin{tabular}{|c|c|c|c|c|c|c|c|c|c|c|c|c|c|c|c|}
\hline Model & 1 & 2 & 3 & 4 & 5 & 6 & 7 & 8 & 9 & 10 & 11 & 12 & 13 & 14 & 15 \\
\hline SK & 1 & 2 & 5 & 8 & 12 & 16 & 26 & 36 & 41 & 46 & 51 & 55 & 58 & 61 & 66 \\
\hline TK1 & 0 & 0 & 1 & 2 & 3 & 6 & 10 & 13 & 16 & 23 & 32 & 38 & 41 & 45 & 49 \\
\hline TK2 & 0 & 0 & 0 & 0 & 1 & 2 & 3 & 6 & 9 & 12 & 16 & 21 & 25 & 31 & 35 \\
\hline TK3 & 0 & 0 & 0 & 0 & 0 & 0 & 1 & 2 & 3 & 6 & 10 & 13 & 16 & 19 & 24 \\
\hline SK Lin & 1 & 2 & 5 & 8 & 13 & 19 & 25 & 32 & 38 & 43 & 48 & 52 & 55 & 58 & 64 \\
\hline Model & 16 & 17 & 18 & 19 & 20 & 21 & 22 & 23 & 24 & 25 & 26 & 27 & 28 & 29 & 30 \\
\hline SK & 75 & 82 & 88 & 92 & 96 & 102 & 108 & $112 \star$ & $116 \star$ & $124 \star$ & $128 \star$ & $132 \star$ & $136 \star$ & $142 \star$ & $148 \star$ \\
\hline TK1 & 54 & 59 & 62 & 66 & 70 & 75 & 79 & 83 & 85 & 88 & 95 & 102 & (108) & (112) & $(120)$ \\
\hline TK2 & 40 & 43 & 47 & 52 & 57 & 59 & 64 & 67 & 72 & 75 & 82 & 85 & 88 & 92 & 96 \\
\hline TK3 & 27 & 31 & 35 & 43 & 45 & 48 & 51 & 55 & 58 & 60 & 65 & 72 & 77 & 81 & 85 \\
\hline SK Lin & 70 & 76 & 80 & 85 & 90 & 96 & 102 & 107 & $110 \dagger$ & $115 \dagger$ & $121 \dagger$ & $127 \dagger$ & $130 \dagger$ & $135 \dagger$ & t \\
\hline
\end{tabular}

\subsection{Other Attacks}

In the original design document $\left[\mathrm{BJK}^{+} 16 \mathrm{a}, \mathrm{BJK}^{+} 16 \mathrm{~b}\right]$, we also analyzed the security of SKINNY with regard to meet-in-the-middle attacks, impossible differential attacks, integral attacks, slide attacks, invariant subspace cryptanalysis, and algebraic attacks. We provide a brief summary of the results and refer the reader to the original documents for details.

\subsubsection{Meet-in-the-Middle Attacks}

We used the property that full diffusion is achieved after six rounds (in both directions) to estimate that meet-in-the middle attacks might work up to at most 22 rounds.

\subsubsection{Impossible Differential Attacks}

We constructed an 11-round truncated impossible differential which can be used for a 16-round key-recovery attack on SKINNY members with a block size of 128 bit with data, time, and memory complexities of $2^{88.5}$ in the single-key model.

\subsubsection{Integral Attacks}

We constructed a 10-round integral distinguisher and used it for a 14-round key-recovery attack.

\subsubsection{Slide Attacks}

The distinction between the rounds is ensured by the round constants and thus the straightforward slide attacks cannot be applied. However, due to the small state of the LFSR, round constants can collide in different rounds.

We took into account all possible sliding numbers of rounds and deduced what is the difference in the constants that is obtained every time. As these constant differences might impact the best differential characteristic, we experimentally checked the lower bounds on the number of active Sboxes for all these constant differences by using MILP. 
In the single-key setting, by allowing any starting round for each value of the slid pair, the lower bounds on the number of active Sboxes reach 36 after 11 rounds, and 41 after 12 rounds. We thus expect that slide attacks do not threaten the security of SKINNY.

\subsubsection{Invariant Subspace Attacks}

The non-trivial key schedule already provides a good protection against such attacks for a larger number of rounds. The main concern that remains are large-dimensional subspaces that propagate invariant through the Sbox. We checked that no such invariant subspaces exist. Moreover, we computed all affine subspaces of dimension larger than two that get mapped to (different) affine subspaces and checked if those can be chained to what could be coined a subspace characteristic. It turns out that those subspaces can be chained only for a very small number of rounds. To conclude, the non-trivial key schedule and the use of round-constants seem to sufficiently protect SKINNY against those attacks.

\subsubsection{Algebraic Attacks}

The Sbox $\mathcal{S}_{8}$ of SKINNY members with a block size of 128 bit has an algebraic degree of 6 and thus, algebraic attacks do not seem to be a threat.

\subsection{Third-Party Cryptanalysis}

Since the publication of the cipher in 2016, there has been lots of cryptanalysis by external researchers. ${ }^{3}$ To the best of our knowledge, we provide a complete list of formally published papers (in the English language) related to mathematical cryptanalysis of SKINNY, as of February 2020. We found 30 such papers in total.

14 of those, namely $\left[\mathrm{ABC}^{+} 17, \mathrm{ZR} 18, \mathrm{EKKT18,} \mathrm{AK18,} \mathrm{SGL}{ }^{+} 17, \mathrm{HV} 18, \mathrm{BCLR}_{17}, \mathrm{ST} 17\right.$, PN17,ZZ18, ZW16, YQC17, $\mathrm{ADG}^{+}$19, DLU19], only consider the variants of SKINNY with a block size of 64 bit or SKINNY-128-128 and we do not mention their results here. We briefly mention the results of the remaining 16 papers in the following.

In [LGS17], the authors conduct cryptanalysis on various variants of SKINNY in the related-tweakey model. For SKINNY-128-256 (resp. SKINNY-128-384), they obtain a 23round (resp. 27-round) related-tweakey impossible differential attack with time complexity $2^{251.47}$ (resp. $2^{378}$ ), data of $2^{124.47}$ (resp. $2^{126.03}$ ) chosen plaintexts and $2^{248}$ (resp. $2^{368}$ ) memory. The impossible differential attack uses a truncated related-tweakey impossible differential over 12 rounds (resp. 16 rounds for the impossible differential attack on SKINNY-128-384). Those complexities were improved under the assumption that the public tweak is loaded in TK-1. For SKINNY-128-384 (resp. SKINNY-128-256), they obtain a 27-round (resp. 22-round) related-tweakey rectangle attack with time complexity $2^{331}$ (resp. $2^{251.03}$ ), data of $2^{112}$ (resp. $2^{118.92}$ ) chosen plaintexts and $2^{144}$ (resp. $2^{120}$ ) memory. The rectangle attacks use actual differential trails with their exact probability. The results indicate that the actual probability of the best differential trails gets lower than estimated by the number of active Sboxes as the number of rounds increases.

In [SMB18], the authors analyze different SKINNY variants with regard to zero-correlation and related-tweakey impossible differential attacks. For SKINNY-128-256, they obtain a related-tweakey impossible differential attack on 23 rounds with time complexity of $2^{243.41}$, data of $2^{124.41}$ chosen plaintexts and $2^{155.41}$ memory. They utilize a 15 -round related-tweakey impossible differential.

In [TAY17], the authors apply impossible differential cryptanalysis on SKINNY in the single-key model. They utilize the 11-round impossible differential described in the design

\footnotetext{
${ }^{3}$ To encourage third-party analysis, we organized three cryptanalysis competitions for SKINNY during the last years. Details can be found at https://sites.google.com/site/skinnycipher/ cryptanalysis-competition.
} 
document. They obtain a key-recovery attack of 20 rounds SKINNY-128-256 with time complexity $2^{245.72}$, data of $2^{92.1}$ chosen plaintexts and memory $2^{147.1}$. They further attack 22 rounds of SKINNY-128-384 with time complexity $2^{373.48}$, data $2^{92.22}$ and memory $2^{147.22}$.

In $\left[\mathrm{SSD}^{+} 18\right]$, the authors used constrained programming for applying the Demirci-Selcuk meet-in-the-middle attack. The authors find an 10.5-round distinguisher and a 22-round key-recovery attack on SKINNY-128-384 with time complexity $2^{382.46}$, data complexity of $2^{96}$ chosen plaintexts and memory complexity of $2^{330.99}$. In [CSSH19], the authors derived a 22-round Demirci-Selcuk meet-in-the-middle key-recovery attack with a reduced time complexity of $2^{366.28}$ by using a key-bridging technique.

In $\left[\mathrm{CHP}^{+} 18\right]$, the authors introduce the Boomerang Connectivity Table (BCT) that quantify the boomerang switching effect in Sboxes wit regard to the boomerang attack. They apply their method to SKINNY and show that the probabilities of the attacks presented in [LGS17] are not precise. Later in [SQH19], the authors re-evaluated the probabilities of the boomerang dstinguishers from [LGS17] using a generalized framework for the BCT. In [WP19], a 4-round boomerang distinguisher on SKINNY with probability 1 is presented.

In $\left[\mathrm{AST}^{+} 17\right]$, the authors proposed a method to model the actual DDT of large Sboxes in order to compute exact probabilities of differential trails. Applied to SKINNY members with a block size of 128 bit, the authors showed that the probability of any 14-round (single-key) differential trail is upper bounded by $2^{-128}$, while the designers proved a lower bound of 61 active Sboxes (ensuring only a probability upper bounded by $2^{-122}$ ).

In [LTW18], the authors present algorithms for finding subspace trails. They find 5 -round subspace trails for all SKINNY members.

In $\left[\mathrm{ABI}^{+} 18\right]$, the authors conduct an exhaustive search over all possible word permutations to be used as a replacement for the ShiftRows permutation and derived the minimum number of active Sboxes with regard to differential cryptanalysis using Matsui's branch-and-bound algorithm. Their results show that the ShiftRows permutation used in SKINNY is actually among the best permutations. By using Matsui's algorithm, they computed the bounds for up to 40 rounds in the single-key setting, while the designers only gave bounds for up to 22 rounds. Table 7 is updated with their improved bounds starting from 23 rounds.

In [BCC19], the authors present a new framework for studying mixture-differential distinguishers and the multiple-of- 8 property, two kind of distinguishers that were recently introduced to distinguish round-reduced versions of the AES. The authors analyze AESlike SPN ciphers with regard to those properties and show that similar results as for the AES can be obtained even for AES-like ciphers for which the MixColumns operation has non-optimal branch number. Applied to SKINNY, the authors show that 5-round SKINNY has the multiple-of- $2^{h}$ property, where $h \in\{1,2, \ldots, 11,13\}$.

In [BGLS19], the authors present a tool, called PEIGEN, for evaluating cryptographic properties of Sboxes. They analyzed the Sbox of SKINNY-128 and found out that it has 601 linear structures and that it is $(7,2)$-linear and $(3,7)$-linear, meaning that $2^{2}$ components are affine on all cosets of a certain 7 -dimensional linear subspace, resp., $2^{7}$ components are affine on all cosets of a certain 3-dimensional linear subspace.

In [KB19], the authors experimentally evaluated the diffusion properties and the indices of strong nonlinearity of the round functions of some lightweight block ciphers, including SKINNY.

In [ZCGP20], the authors introduce a new method for checking the resistance of an SPN cipher against integral distinguishers, truncated differentials and impossible differential distinguishers in the single-key setting by considering an algebraic representation of the round function and analyzing its structure. They could find several 10-round integral distinguishers, the 11-round impossible differential distinguishers, and a 7-round truncated differential distinguisher. They further analyze SKINNY-128-128 explicitly and provide a practical 11-round attack in the single-key model. 
In $\left[\mathrm{ZDM}^{+} 20\right]$, the authors provide a new key-recovery model of related-key rectangle attacks on block ciphers with linear key schedules. Applying their technique to SKINNY, the authors obtain a 28-round related-tweakey rectangle attack on SKINNY-128-384 with time complexity $2^{315.25}$, data of $2^{122}$ chosen plaintexts and memory $2^{122.32}$.

As a summary, Table 8 shows the maximum number of rounds that can be attacked so far. Both of the underlying primitives SKINNY-128-256 and SKINNY-128-384 still offer a security margin of at least $50 \%$.

Table 8: Number of rounds of SKINNY that can be attacked by the best key-recovery attacks (in the related-tweakey model) known so far.

\begin{tabular}{cc}
\hline SKINNY-128-256 & SKINNY-128-384 \\
\hline $23 / 48$ & $28 / 56$ \\
$47.9 \%$ & $50.0 \%$ \\
\hline
\end{tabular}

Remark on security margin. We would like to emphasise that Table 8 reflects the security margin of the underlying tweakable block cipher SKINNY-128-256 and SKINNY-128-384. In the context of SKINNY-AEAD and SKINNY-Hash, most of the attack model does not hold (for example due to data limit or limited control over the input to the TBC) and the attacks are not applicable to SKINNY-AEAD or SKINNY-Hash that uses the same reduced-round SKINNY. For example, having a theoretical attack on 28-round SKINNY-128-384 does not imply an attack on SKINNY-AEAD or SKINNY-Hash that uses 28-round SKINNY-128-384.

\section{Hardware Implementations}

We also provide performance results and area footprints of SKINNY-AEAD and SKINNY-Hash when implemented on a hardware platform. To this end, in addition to the tk3 and tk2 constructions of SKINNY-Hash, we realized two sets of implementations for each SKINNY-AEAD variant: one as encryption-only and the other one supporting both encryption and decryption functionalities. We further considered two different instances of the underlying SKINNY module: a round-based implementation performing every cipher round in a clock cycle, and a byte-serial implementation mainly processing a single byte per clock cycle. The corresponding results are depicted in Table 9 and Table 11, respectively, where the area footprint (in GE), maximum clock frequency, and maximum throughput for two standard cell libraries IBM 130 and UMC 90 are reported. In order to achieve the maximum throughput, we simulated the SKINNY-AEAD implementations with 100 blocks of 16-byte associated data $A$ and 100 blocks of 16 -byte message $M$, thereby obtaining the required number of clock cycles. Note that the number of clock cycles is independent of the value of the given associated data and the message as our implementations are constant-time preventing any leakage through the timing side channel. For SKINNY-Hash we obtained the number of required clock cycles by simulating the implementation with a message of 100 blocks of 16-byte (for SKINNY-tk3-Hash) and a message of 100 blocks of 4-byte (for SKINNY-tk2-Hash).

We further realized the side-channel protected variant of all aforementioned implementations. We applied a masking countermeasure with 2 shares and made use of the concept explained in $\left[\mathrm{RBN}^{+} 15\right]$, i.e., how to achieve $d$ th order security using $d+1$ shares. It is noteworthy that due to the special construction of the SKINNY Sbox, its SCA-protected version with 2 shares does not require any fresh randomness. Similar observations have been reported in $\left[\mathrm{RBN}^{+} 15\right]$ and $\left[\mathrm{DDE}^{+} 19\right]$, where particular functions (mainly made by a combination of AND-XOR) can be uniformly shared without any fresh masks. Therefore, in all variants of SKINNY-AEAD, it is sufficient to present the entire inputs and output 
(including the associated data, message, key, tag and output) by two uniformly-masked additive shares. It also holds for SKINNY-Hash, while SKINNY-tk3-Hash requires an additional 384-bit of initial mask used to initialize the state $S_{384}$ in a shared way with 2 shares. Trivially, SKINNY-tk3-Hash also needs such an initial mask (of 256 bits). The performance results and the area footprint of all implementations are given in Table 10 and Table 12. The particular implementations can be found in the supplementary material and at https://sites.google.com/site/skinnycipher/downloads.

Table 9: Unprotected, round-based ASIC implementations of our SKINNY-AEAD and SKINNY-Hash members using IBM 130 and UMC 90 standard cell libraries.

\begin{tabular}{lccc|ccc}
\hline & \multicolumn{3}{c}{ IBM 130 } & \multicolumn{3}{c}{ UMC 90 } \\
\cline { 2 - 7 } & Area & Freq. & Throughput & Area & Freq. & Throughput \\
\cline { 2 - 7 } & GE & MHz & MBit/s & GE & MHz & MBit/s \\
\hline SKINNY-AEAD-M1-Enc & 7627 & 184 & 406 & 7808 & 269 & 594 \\
SKINNY-AEAD-M2-Enc & 7595 & 181 & 400 & 7808 & 287 & 633 \\
SKINNY-AEAD-M3-Enc & 7100 & 189 & 418 & 7291 & 267 & 589 \\
SKINNY-AEAD-M4-Enc & 7069 & 164 & 363 & 7266 & 233 & 514 \\
SKINNY-AEAD-M5-Enc & 6512 & 171 & 428 & 6636 & 298 & 743 \\
SKINNY-AEAD-M6-Enc & 5953 & 208 & 519 & 6099 & 288 & 720 \\
\hline SKINNY-AEAD-M1-Enc-Dec & 10370 & 158 & 349 & 10239 & 227 & 501 \\
SKINNY-AEAD-M2-Enc-Dec & 10318 & 166 & 367 & 10210 & 240 & 530 \\
SKINNY-AEAD-M3-Enc-Dec & 9817 & 167 & 368 & 9671 & 244 & 539 \\
SKINNY-AEAD-M4-Enc-Dec & 9772 & 164 & 362 & 9564 & 202 & 447 \\
SKINNY-AEAD-M5-Enc-Dec & 8844 & 166 & 416 & 8894 & 268 & 670 \\
SKINNY-AEAD-M6-Enc-Dec & 8290 & 166 & 414 & 8332 & 222 & 555 \\
\hline SKINNY-tk3-Hash & 8622 & 212 & 154 & 8894 & 285 & 207 \\
SKINNY-tk2-Hash & 5730 & 211 & 66 & 6019 & 304 & 95 \\
\hline
\end{tabular}

Table 10: SCA-protected (2 shares), round-based ASIC implementations of SKINNY-AEAD and SKINNY-Hash members using IBM 130 and UMC 90 standard cell libraries.

\begin{tabular}{lccc|ccc}
\hline & \multicolumn{3}{c}{ IBM 130 } & \multicolumn{3}{c}{ UMC 90 } \\
\cline { 2 - 5 } & Area & Freq. & Throughput & Area & Freq. & Throughput \\
\cline { 2 - 3 } \cline { 5 - 7 } & GE & MHz & MBit/s & GE & MHz & MBit/s \\
\hline SKINNY-AEAD-M1-Enc & 13812 & 427 & 192 & 14481 & 885 & 398 \\
SKINNY-AEAD-M2-Enc & 13787 & 427 & 192 & 14445 & 885 & 398 \\
SKINNY-AEAD-M3-Enc & 12716 & 427 & 192 & 13422 & 885 & 398 \\
SKINNY-AEAD-M4-Enc & 12692 & 427 & 192 & 13383 & 885 & 398 \\
SKINNY-AEAD-M5-Enc & 12653 & 435 & 228 & 13263 & 885 & 464 \\
SKINNY-AEAD-M6-Enc & 11555 & 422 & 221 & 12203 & 885 & 464 \\
\hline SKINNY-AEAD-M1-Enc-Dec & 18817 & 446 & 201 & 20534 & 909 & 409 \\
SKINNY-AEAD-M2-Enc-Dec & 18768 & 444 & 200 & 20460 & 917 & 413 \\
SKINNY-AEAD-M3-Enc-Dec & 17723 & 446 & 201 & 19474 & 909 & 409 \\
SKINNY-AEAD-M4-Enc-Dec & 17675 & 444 & 200 & 19400 & 917 & 413 \\
SKINNY-AEAD-M5-Enc-Dec & 17285 & 435 & 228 & 18888 & 820 & 430 \\
SKINNY-AEAD-M6-Enc-Dec & 16180 & 448 & 235 & 17828 & 820 & 430 \\
\hline SKINNY-tk3-Hash & 18388 & 429 & 64 & 19178 & 787 & 118 \\
SKINNY-tk2-Hash & 12404 & 405 & 26 & 13041 & 794 & 52 \\
\hline
\end{tabular}

\section{New Variants}

As shown in Section 5.3, the SKINNY tweakable block cipher went through a lot of third-party analysis efforts already and still has a very large security margin at time of writing. The best known attacks against SKINNY-128-256 cover only 23 rounds [LGS17, SMB18] (out of 48 rounds), while the best attack against SKINNY-128-384 covers 28 rounds [ZDM $\left.{ }^{+} 20\right]$ 
Table 11: Unprotected, byte-serial ASIC implementations of SKINNY-AEAD/ SKINNY-Hash.

\begin{tabular}{lccc|ccc}
\hline & \multicolumn{3}{c}{ IBM 130 } & \multicolumn{3}{c}{ UMC 90 } \\
\cline { 2 - 7 } & Area & Freq. & Throughput & Area & Freq. & Throughput \\
\cline { 2 - 7 } & GE & MHz & MBit/s & GE & MHz & MBit/s \\
\hline SKINNY-AEAD-M1-Enc & 7270 & 532 & 57 & 7253 & 1124 & 121 \\
SKINNY-AEAD-M2-Enc & 7238 & 532 & 57 & 7237 & 1124 & 121 \\
SKINNY-AEAD-M3-Enc & 6723 & 418 & 45 & 6709 & 654 & 71 \\
SKINNY-AEAD-M4-Enc & 6690 & 418 & 45 & 6707 & 654 & 71 \\
SKINNY-AEAD-M5-Enc & 6157 & 439 & 55 & 6199 & 1429 & 180 \\
SKINNY-AEAD-M6-Enc & 5601 & 478 & 60 & 5669 & 1429 & 180 \\
\hline SKINNY-AEAD-M1-Enc-Dec & 9554 & 291 & 31 & 9038 & 327 & 35 \\
SKINNY-AEAD-M2-Enc-Dec & 9485 & 228 & 25 & 8939 & 340 & 37 \\
SKINNY-AEAD-M3-Enc-Dec & 9002 & 284 & 31 & 8516 & 392 & 42 \\
SKINNY-AEAD-M4-Enc-Dec & 8947 & 258 & 28 & 8428 & 524 & 57 \\
SKINNY-AEAD-M5-Enc-Dec & 8002 & 264 & 33 & 7702 & 412 & 52 \\
SKINNY-AEAD-M6-Enc-Dec & 7456 & 267 & 34 & 7179 & 422 & 53 \\
\hline SKINNY-tk3-Hash & 8406 & 485 & 17 & 8405 & 741 & 26 \\
SKINNY-tk2-Hash & 5554 & 402 & 6 & 5484 & 538 & 8 \\
\hline
\end{tabular}

Table 12: SCA-protected (2 shares), byte-serial ASIC implementations of SKINNY-AEAD and SKINNY-Hash members.

\begin{tabular}{lccc|ccc}
\hline & \multicolumn{3}{c}{ IBM 130 } & \multicolumn{3}{c}{ UMC 90 } \\
\cline { 2 - 7 } & Area & Freq. & Throughput & Area & Freq. & Throughput \\
\cline { 2 - 6 } \cline { 4 - 7 } & GE & MHz & MBit/s & GE & MHz & MBit/s \\
\hline SKINNY-AEAD-M1-Enc & 13289 & 287 & 8 & 13939 & 424 & 11 \\
SKINNY-AEAD-M2-Enc & 13265 & 287 & 8 & 13913 & 424 & 11 \\
SKINNY-AEAD-M3-Enc & 12195 & 287 & 8 & 12881 & 424 & 11 \\
SKINNY-AEAD-M4-Enc & 12171 & 287 & 8 & 12852 & 424 & 11 \\
SKINNY-AEAD-M5-Enc & 12143 & 274 & 9 & 12756 & 448 & 14 \\
SKINNY-AEAD-M6-Enc & 11048 & 274 & 9 & 11694 & 448 & 14 \\
\hline SKINNY-AEAD-M1-Enc-Dec & 17115 & 342 & 9 & 18452 & 478 & 13 \\
SKINNY-AEAD-M2-Enc-Dec & 17065 & 342 & 9 & 18369 & 478 & 13 \\
SKINNY-AEAD-M3-Enc-Dec & 16019 & 342 & 9 & 17394 & 478 & 13 \\
SKINNY-AEAD-M4-Enc-Dec & 15968 & 342 & 9 & 17309 & 478 & 13 \\
SKINNY-AEAD-M5-Enc-Dec & 15528 & 248 & 8 & 16719 & 610 & 19 \\
SKINNY-AEAD-M6-Enc-Dec & 14432 & 248 & 8 & 15660 & 610 & 19 \\
\hline SKINNY-tk3-Hash & 17698 & 265 & 2 & 18793 & 505 & 4 \\
SKINNY-tk2-Hash & 11827 & 292 & 1 & 12514 & 348 & 1 \\
\hline
\end{tabular}

(out of 56 rounds). All these attacks have very high complexity, much more than $2^{200}$ in computational complexity and sometimes up to almost $2^{384}$, and only work in the related-tweakey model where differences need to also be inserted in the tweak and/or key input. This means that for both SKINNY-128-256 and SKINNY-128-384 versions, the security margin is at least of $50 \%$ and actually much more if one considers only single-key attacks and/or attacks with a complexity lower than $2^{128}$ (in the single-key model, the best known attacks against SKINNY-128-256 cover only 20 rounds [TAY17], while the best attack against SKINNY-128-384 covers 22 rounds [TAY17, $\left.\mathrm{SSD}^{+} 18, \mathrm{CSSH} 19\right]$, again all these attacks having a very high computational complexity).

Most block ciphers have a security margin usually at very best around $20 / 30 \%$. This indicates that a $50 \%$ security margin may be overly large. For this reason, we naturally propose new variants for the versions of SKINNY-AEAD and SKINNY-Hash based on SKINNY-128-384: SKINNY-AEAD-M1+, SKINNY-AEAD-M2+, SKINNY-AEAD-M3+, SKINNY-AEAD-M4+ as well as SKINNY-tk3-Hash+. These members share exactly the same specifications as SKINNY-AEAD-M1, SKINNY-AEAD-M2, SKINNY-AEAD-M3, SKINNY-AEAD-M4 and SKINNY-tk3-Hash, except that the number of SKINNY-128-384 rounds is reduced from 56 to 40 to give a very comfortable 
security margin of around 30\% (in the worst-case related-tweakey scenario, without even excluding attacks with complexity significantly higher than $2^{128}$ ). The security claims of these new members are exactly the same as the security claims of the old members they are based on, but they are expected to be around $1.4 \mathrm{x}$ faster than their counterparts for the same area cost.

\section{Acknowledgements}

The work described in this paper has been supported in part by the Deutsche Forschungsgemeinschaft (DFG, German Research Foundation) under Germany's Excellence Strategy - EXC 2092 CASA - 390781972.

\section{References}

$\left[\mathrm{ABC}^{+} 17\right]$ Ralph Ankele, Subhadeep Banik, Avik Chakraborti, Eik List, Florian Mendel, Siang Meng Sim, and Gaoli Wang. Related-key impossible-differential attack on reduced-round Skinny. In Dieter Gollmann, Atsuko Miyaji, and Hiroaki Kikuchi, editors, Applied Cryptography and Network Security - 15th International Conference, ACNS 2017, Kanazawa, Japan, July 10-12, 2017, Proceedings, volume 10355 of LNCS, pages 208-228. Springer, 2017.

[ABI $\left.{ }^{+} 18\right]$ Gianira N. Alfarano, Christof Beierle, Takanori Isobe, Stefan Kölbl, and Gregor Leander. ShiftRows alternatives for AES-like ciphers and optimal cell permutations for Midori and Skinny. IACR Trans. Symmetric Cryptol., 2018(2):20-47, 2018.

[ADG $\left.{ }^{+} 19\right]$ Ralph Ankele, Christoph Dobraunig, Jian Guo, Eran Lambooij, Gregor Leander, and Yosuke Todo. Zero-correlation attacks on tweakable block ciphers with linear tweakey expansion. IACR Trans. Symmetric Cryptol., 2019(1):192-235, 2019.

[AK18] Ralph Ankele and Stefan Kölbl. Mind the gap - A closer look at the security of block ciphers against differential cryptanalysis. In Cid and Jacobson Jr. [CJ19], pages $163-190$.

$\left[\mathrm{AST}^{+}\right.$17] Ahmed Abdelkhalek, Yu Sasaki, Yosuke Todo, Mohamed Tolba, and Amr M. Youssef. MILP modeling for (large) s-boxes to optimize probability of differential characteristics. IACR Trans. Symmetric Cryptol., 2017(4):99-129, 2017.

[BCC19] Christina Boura, Anne Canteaut, and Daniel Coggia. A general proof framework for recent AES distinguishers. IACR Trans. Symmetric Cryptol., 2019(1):170-191, 2019.

[BCLR17] Christof Beierle, Anne Canteaut, Gregor Leander, and Yann Rotella. Proving resistance against invariant attacks: How to choose the round constants. In Jonathan Katz and Hovav Shacham, editors, Advances in Cryptology CRYPTO 2017 - 37th Annual International Cryptology Conference, Santa Barbara, CA, USA, August 20-24, 2017, Proceedings, Part II, volume 10402 of $L N C S$, pages 647-678. Springer, 2017.

[BDPA11] Guido Bertoni, Joan Daemen, Michaël Peeters, and Gilles Van Assche. Cryptographic sponge functions. http://sponge.noekeon.org/, 2011. 
[BDPVA07] Guido Bertoni, Joan Daemen, Michaël Peeters, and Gilles Van Assche. Sponge functions. In ECRYPT hash workshop, 2007.

[BGLS19] Zhenzhen Bao, Jian Guo, San Ling, and Yu Sasaki. PEIGEN - a platform for evaluation, implementation, and generation of S-boxes. IACR Trans. Symmetric Cryptol., 2019(1):330-394, 2019.

$\left[\mathrm{BJK}^{+} 16 \mathrm{a}\right]$ Christof Beierle, Jérémy Jean, Stefan Kölbl, Gregor Leander, Amir Moradi, Thomas Peyrin, Yu Sasaki, Pascal Sasdrich, and Siang Meng Sim. The SKINNY family of block ciphers and its low-latency variant MANTIS. In Matthew Robshaw and Jonathan Katz, editors, Advances in Cryptology CRYPTO 2016 - 36th Annual International Cryptology Conference, Santa Barbara, CA, USA, August 14-18, 2016, Proceedings, Part II, volume 9815 of LNCS, pages 123-153. Springer, 2016.

$\left[\mathrm{BJK}^{+} 16 \mathrm{~b}\right]$ Christof Beierle, Jérémy Jean, Stefan Kölbl, Gregor Leander, Amir Moradi, Thomas Peyrin, Yu Sasaki, Pascal Sasdrich, and Siang Meng Sim. The SKINNY family of block ciphers and its low-latency variant MANTIS. IACR Cryptology ePrint Archive, 2016:660, 2016.

[BS90] Eli Biham and Adi Shamir. Differential cryptanalysis of des-like cryptosystems. In Alfred Menezes and Scott A. Vanstone, editors, Advances in Cryptology - CRYPTO '90, 10th Annual International Cryptology Conference, Santa Barbara, California, USA, August 11-15, 1990, Proceedings, volume 537 of $L N C S$, pages 2-21. Springer, 1990.

$\left[\mathrm{CHP}^{+}{ }^{18}\right]$ Carlos Cid, Tao Huang, Thomas Peyrin, Yu Sasaki, and Ling Song. Boomerang connectivity table: A new cryptanalysis tool. In Jesper Buus Nielsen and Vincent Rijmen, editors, Advances in Cryptology - EUROCRYPT 2018 37th Annual International Conference on the Theory and Applications of Cryptographic Techniques, Tel Aviv, Israel, April 29 - May 3, 2018 Proceedings, Part II, volume 10821 of $L N C S$, pages 683-714. Springer, 2018.

[CJ19] Carlos Cid and Michael J. Jacobson Jr., editors. Selected Areas in Cryptography - SAC 2018 - 25th International Conference, Calgary, AB, Canada, August 15-17, 2018, Revised Selected Papers, volume 11349 of LNCS. Springer, 2019.

[CSSH19] Qiu Chen, Danping Shi, Siwei Sun, and Lei Hu. Automatic demirci-selçuk meet-in-the-middle attack on SKINNY with key-bridging. In Jianying Zhou, Xiapu Luo, Qingni Shen, and Zhen Xu, editors, Information and Communications Security - 21st International Conference, ICICS 2019, Beijing, China, December 15-17, 2019, Revised Selected Papers, volume 11999 of LNCS, pages 233-247. Springer, 2019.

$\left[\mathrm{DDE}^{+} 19\right]$ Joan Daemen, Christoph Dobraunig, Maria Eichlseder, Hannes Groß, Florian Mendel, and Robert Primas. Protecting against statistical ineffective fault attacks. IACR Cryptology ePrint Archive, 2019:536, 2019.

[DLU19] Patrick Derbez, Virginie Lallemand, and Aleksei Udovenko. Cryptanalysis of SKINNY in the framework of the SKINNY 2018-2019 cryptanalysis competition. In Kenneth G. Paterson and Douglas Stebila, editors, Selected Areas in Cryptography - SAC 2019 - 26th International Conference, Waterloo, ON, Canada, August 12-16, 2019, Revised Selected Papers, volume 11959 of LNCS, pages 124-145. Springer, 2019.

[Dwo15] Morris J Dworkin. SHA-3 standard: Permutation-based hash and extendableoutput functions. Federal Inf. Process. Stds.(NIST FIPS)-202, 2015. 
[EKKT18] Zahra Eskandari, Andreas Brasen Kidmose, Stefan Kölbl, and Tyge Tiessen. Finding integral distinguishers with ease. In Cid and Jacobson Jr. [CJ19], pages 115-138.

[HV18] Mathias Hall-Andersen and Philip S. Vejre. Generating graphs packed with paths. IACR Trans. Symmetric Cryptol., 2018(3):265-289, 2018.

[JNP14] Jérémy Jean, Ivica Nikolic, and Thomas Peyrin. Tweaks and keys for block ciphers: The TWEAKEY framework. In Palash Sarkar and Tetsu Iwata, editors, Advances in Cryptology - ASIACRYPT 2014 - 20th International Conference on the Theory and Application of Cryptology and Information Security, Kaoshiung, Taiwan, R.O.C., December 7-11, 2014, Proceedings, Part II, volume 8874 of $L N C S$, pages 274-288. Springer, 2014.

[JNPS16] Jérémy Jean, Ivica Nikolić, Thomas Peyrin, and Yannick Seurin. Deoxys v1.41, 2016. Submission to CAESAR, available via https://competitions . cr.yp.to/round3/deoxysv141.pdf.

[KB19] I. Khairullin and V. Bobrov. On cryptographic properties of some lightweight algorithms and its application to the construction of s-boxes. In 2019 IEEE Conference of Russian Young Researchers in Electrical and Electronic Engineering (EIConRus), pages 1807-1810, 2019.

[KLW17] Thorsten Kranz, Gregor Leander, and Friedrich Wiemer. Linear cryptanalysis: Key schedules and tweakable block ciphers. IACR Trans. Symmetric Cryptol., 2017(1):474-505, 2017.

[KPPY14] Khoongming Khoo, Thomas Peyrin, Axel York Poschmann, and Huihui Yap. FOAM: searching for hardware-optimal SPN structures and components with a fair comparison. In Lejla Batina and Matthew Robshaw, editors, Cryptographic Hardware and Embedded Systems - CHES 2014 - 16th International Workshop, Busan, South Korea, September 23-26, 2014. Proceedings, volume 8731 of $L N C S$, pages 433-450. Springer, 2014.

[KR11] Ted Krovetz and Phillip Rogaway. The software performance of authenticatedencryption modes. In Antoine Joux, editor, Fast Software Encryption - 18th International Workshop, FSE 2011, Lyngby, Denmark, February 13-16, 2011, Revised Selected Papers, volume 6733 of Lecture Notes in Computer Science, pages 306-327. Springer, 2011.

[KR16] Ted Krovetz and Phillip Rogaway. OCB (v1.1), 2016. Submission to CAESAR, available via https://competitions.cr.yp.to/round3/ocbv11.pdf.

[KS05] John Kelsey and Bruce Schneier. Second preimages on n-bit hash functions for much less than $2^{\mathrm{n}}$ work. In Ronald Cramer, editor, Advances in Cryptology EUROCRYPT 2005, 24th Annual International Conference on the Theory and Applications of Cryptographic Techniques, Aarhus, Denmark, May 22-26, 2005, Proceedings, volume 3494 of LNCS, pages 474-490. Springer, 2005.

[LGS17] Guozhen Liu, Mohona Ghosh, and Ling Song. Security analysis of SKINNY under related-tweakey settings (long paper). IACR Trans. Symmetric Cryptol., 2017(3):37-72, 2017.

[LRW02] Moses Liskov, Ronald L. Rivest, and David A. Wagner. Tweakable block ciphers. In Moti Yung, editor, Advances in Cryptology - CRYPTO 2002, 22nd Annual International Cryptology Conference, Santa Barbara, California, USA, August 18-22, 2002, Proceedings, volume 2442 of LNCS, pages 31-46. Springer, 2002. 
[LTW18] Gregor Leander, Cihangir Tezcan, and Friedrich Wiemer. Searching for subspace trails and truncated differentials. IACR Trans. Symmetric Cryptol., 2018(1):74-100, 2018.

[Mat93] Mitsuru Matsui. Linear cryptanalysis method for DES cipher. In Tor Helleseth, editor, Advances in Cryptology - EUROCRYPT '93, Workshop on the Theory and Application of of Cryptographic Techniques, Lofthus, Norway, May 23-27, 1993, Proceedings, volume 765 of LNCS, pages 386-397. Springer, 1993.

$\left[\mathrm{MPL}^{+} 11\right]$ Amir Moradi, Axel Poschmann, San Ling, Christof Paar, and Huaxiong Wang. Pushing the limits: A very compact and a threshold implementation of AES. In Kenneth G. Paterson, editor, Advances in Cryptology - EUROCRYPT 2011 - 30th Annual International Conference on the Theory and Applications of Cryptographic Techniques, Tallinn, Estonia, May 15-19, 2011. Proceedings, volume 6632 of $L N C S$, pages 69-88. Springer, 2011.

[MV04] David McGrew and John Viega. The Galois/Counter mode of operation (GCM). Submission to NIST. http://csrc. nist. gov/CryptoToolk it/modes/proposedmodes/gcm/gcm-spec. pdf, 2004.

[MWGP11] Nicky Mouha, Qingju Wang, Dawu Gu, and Bart Preneel. Differential and linear cryptanalysis using mixed-integer linear programming. In Chuankun Wu, Moti Yung, and Dongdai Lin, editors, Information Security and Cryptology 7th International Conference, Inscrypt 2011, Beijing, China, November 30 - December 3, 2011. Revised Selected Papers, volume 7537 of LNCS, pages 57-76. Springer, 2011.

[Nat18] National Institute of Standards and Technology. Announcing request for nominations for lightweight cryptographic algorithms. Federal Register, 83(166):43656-43657, 2018. https://www.govinfo.gov/content/pkg/FR-201808-27/pdf/2018-18433.pdf.

[NO14] Yusuke Naito and Kazuo Ohta. Improved indifferentiable security analysis of PHOTON. In Michel Abdalla and Roberto De Prisco, editors, Security and Cryptography for Networks - 9th International Conference, SCN 2014, Amalfi, Italy, September 3-5, 2014. Proceedings, volume 8642 of LNCS, pages 340-357. Springer, 2014.

[NSS20] Yusuke Naito, Yu Sasaki, and Takeshi Sugawara. Lightweight Authenticated Encryption Mode Suitable for Threshold Implementation. In Anne Canteaut and Yuval Ishai, editors, Advances in Cryptology - EUROCRYPT 2020, 39th Annual International Conference on the Theory and Applications of Cryptographic Techniques, Proceedings, LNCS, Springer, To appear.

[NRS11] Svetla Nikova, Vincent Rijmen, and Martin Schläffer. Secure hardware implementation of nonlinear functions in the presence of glitches. J. Cryptology, 24(2):292-321, 2011.

[PN17] Raluca Posteuca and Gabriel Negara. New related-key attacks and properties of SKINNY-64-128 cipher. Proceedings of the Romanian Academy, Series A, 18, Special Issue 2017:333-350, 2017.

[RBBK01] Phillip Rogaway, Mihir Bellare, John Black, and Ted Krovetz. OCB: a blockcipher mode of operation for efficient authenticated encryption. In Michael K. Reiter and Pierangela Samarati, editors, CCS 2001, Proceedings of the 8th ACM Conference on Computer and Communications Security, Philadelphia, Pennsylvania, USA, November 6-8, 2001., pages 196-205. ACM, 2001. 
$\left[\mathrm{RBN}^{+} 15\right] \quad$ Oscar Reparaz, Begül Bilgin, Svetla Nikova, Benedikt Gierlichs, and Ingrid Verbauwhede. Consolidating masking schemes. In Rosario Gennaro and Matthew Robshaw, editors, Advances in Cryptology - CRYPTO 2015 - 35th Annual Cryptology Conference, Santa Barbara, CA, USA, August 16-20, 2015, Proceedings, Part I, volume 9215 of LNCS, pages 764-783. Springer, 2015.

[Rog04] Phillip Rogaway. Efficient instantiations of tweakable blockciphers and refinements to modes OCB and PMAC. In Pil Joong Lee, editor, Advances in Cryptology - ASIACRYPT 2004, 10th International Conference on the Theory and Application of Cryptology and Information Security, Jeju Island, Korea, December 5-9, 2004, Proceedings, volume 3329 of LNCS, pages 16-31. Springer, 2004.

[Sch98] Rick Schroeppel. The Hasty Pudding Cipher. NIST AES proposal, 1998.

$\left[\mathrm{SGL}^{+} 17\right]$ Siwei Sun, David Gerault, Pascal Lafourcade, Qianqian Yang, Yosuke Todo, Kexin Qiao, and Lei Hu. Analysis of AES, SKINNY, and others with constraint programming. IACR Trans. Symmetric Cryptol., 2017(1):281-306, 2017.

[SHS $\left.{ }^{+} 13\right] \quad$ Siwei Sun, Lei Hu, Ling Song, Yonghong Xie, and Peng Wang. Automatic security evaluation of block ciphers with s-bp structures against related-key differential attacks. In Dongdai Lin, Shouhuai Xu, and Moti Yung, editors, Information Security and Cryptology - 9th International Conference, Inscrypt 2013, Guangzhou, China, November 27-30, 2013, Revised Selected Papers, volume 8567 of $L N C S$, pages 39-51. Springer, 2013.

$\left[\mathrm{SIH}^{+} 11\right] \quad$ Kyoji Shibutani, Takanori Isobe, Harunaga Hiwatari, Atsushi Mitsuda, Toru Akishita, and Taizo Shirai. Piccolo: An ultra-lightweight blockcipher. In Cryptographic Hardware and Embedded Systems - CHES 2011 - 13th International Workshop, Nara, Japan, September 28 - October 1, 2011. Proceedings, volume 6917 of $L N C S$, pages 342-357. Springer, 2011.

[SMB18] Sadegh Sadeghi, Tahereh Mohammadi, and Nasour Bagheri. Cryptanalysis of reduced round SKINNY block cipher. IACR Trans. Symmetric Cryptol., 2018(3):124-162, Sep. 2018.

[SQH19] Ling Song, Xianrui Qin, and Lei Hu. Boomerang connectivity table revisited. application to SKINNY and AES. IACR Trans. Symmetric Cryptol., 2019(1):118-141, 2019.

[SSD ${ }^{+}$18] Danping Shi, Siwei Sun, Patrick Derbez, Yosuke Todo, Bing Sun, and Lei Hu. Programming the demirci-selçuk meet-in-the-middle attack with constraints. In Thomas Peyrin and Steven D. Galbraith, editors, Advances in Cryptology - ASIACRYPT 2018 - 24th International Conference on the Theory and Application of Cryptology and Information Security, Brisbane, QLD, Australia, December 2-6, 2018, Proceedings, Part II, volume 11273 of LNCS, pages 3-34. Springer, 2018.

[ST17] Yu Sasaki and Yosuke Todo. New impossible differential search tool from design and cryptanalysis aspects - revealing structural properties of several ciphers. In Jean-Sébastien Coron and Jesper Buus Nielsen, editors, Advances in Cryptology - EUROCRYPT 2017 - 36th Annual International Conference on the Theory and Applications of Cryptographic Techniques, Paris, France, April 30 - May 4, 2017, Proceedings, Part III, volume 10212 of LNCS, pages 185-215, 2017. 
[TAY17] Mohamed Tolba, Ahmed Abdelkhalek, and Amr M. Youssef. Impossible differential cryptanalysis of reduced-round SKINNY. In Marc Joye and Abderrahmane Nitaj, editors, Progress in Cryptology - AFRICACRYPT 2017 - 9th International Conference on Cryptology in Africa, Dakar, Senegal, May 24-26, 2017, Proceedings, volume 10239 of LNCS, pages 117-134, 2017.

[VV17] Serge Vaudenay and Damian Vizár. Under pressure: Security of caesar candidates beyond their guarantees. IACR Cryptology ePrint Archive, 2017:1147, 2017.

[WP19] Haoyang Wang and Thomas Peyrin. Boomerang switch in multiple rounds. application to AES variants and Deoxys. IACR Trans. Symmetric Cryptol., 2019(1):142-169, 2019.

[YQC17] Dong Yang, Wen-Feng Qi, and Hua-Jin Chen. Impossible differential attacks on the SKINNY family of block ciphers. IET Information Security, 11(6):377$385,2017$.

[ZCGP20] Wenying Zhang, Meichun Cao, Jian Guo, and Enes Pasalic. Improved security evaluation of spn block ciphers and its applications in the single-key attack on SKINNY. IACR Trans. Symmetric Cryptol., 2019(4):171-191, 2020.

$\left[\mathrm{ZDM}^{+} 20\right]$ Boxin Zhao, Xiaoyang Dong, Willi Meier, Keting Jia, and Gaoli Wang. Generalized related-key rectangle attacks on block ciphers with linear key schedule: applications to SKINNY and GIFT. Designs, Codes and Cryptography, pages $1-24,2020$.

[ZR18] Wenying Zhang and Vincent Rijmen. Division cryptanalysis of block ciphers with a binary diffusion layer. IET Information Security, August 2018.

[ZW16] Yafei Zheng and Wenling Wu. Biclique attack of block cipher SKINNY. In Kefei Chen, Dongdai Lin, and Moti Yung, editors, Information Security and Cryptology - 12th International Conference, Inscrypt 2016, Beijing, China, November 4-6, 2016, Revised Selected Papers, volume 10143 of LNCS, pages 3-17. Springer, 2016.

[ZZ18] Pei Zhang and Wenying Zhang. Differential cryptanalysis on block cipher skinny with MILP program. Security and Communication Networks, 2018:3780407:1-3780407:11, 2018. 


\section{A The 8-bit Sbox for SKINNY}

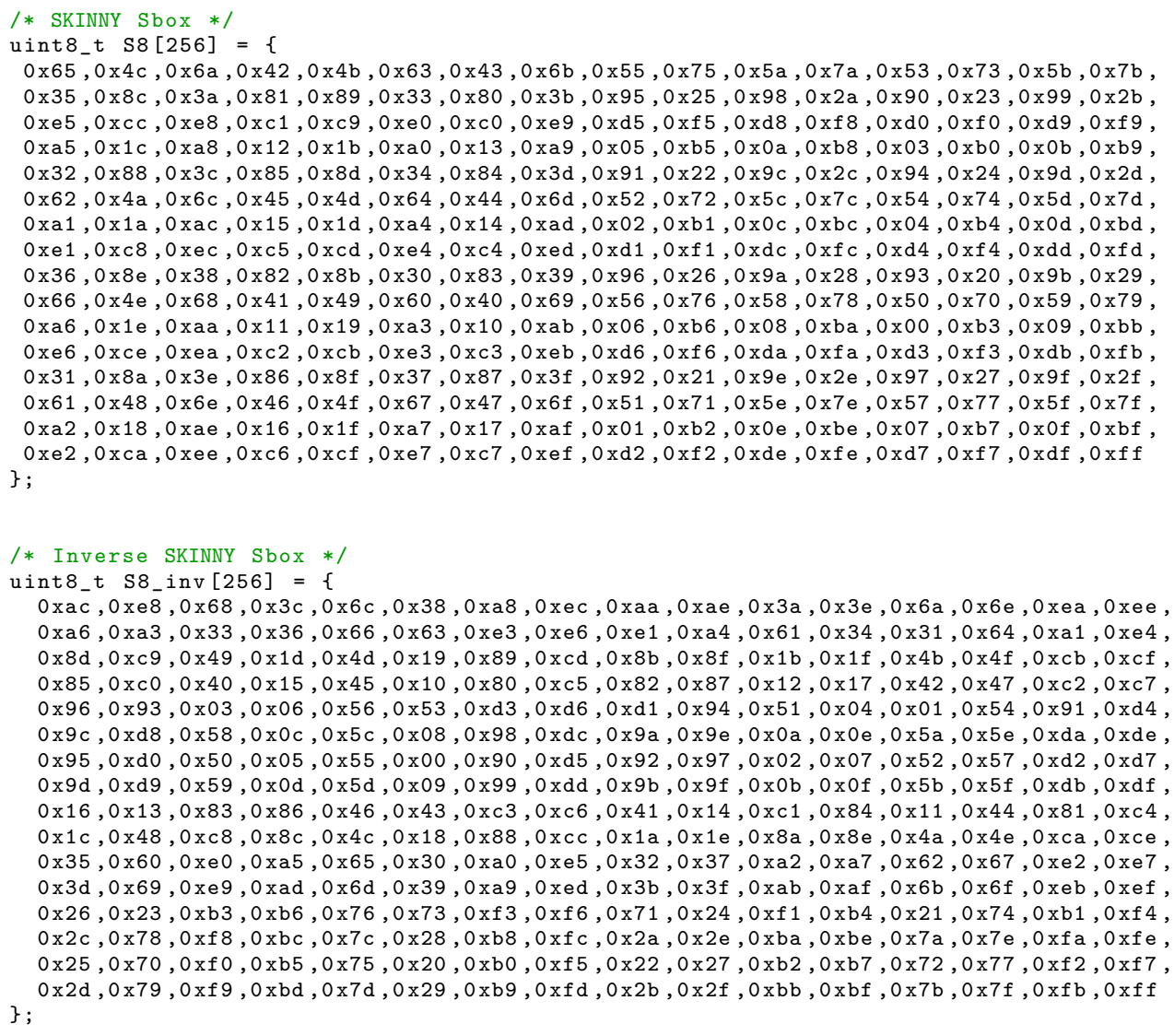

B Test Vectors for SKINNY-128-256 and SKINNY-128-384

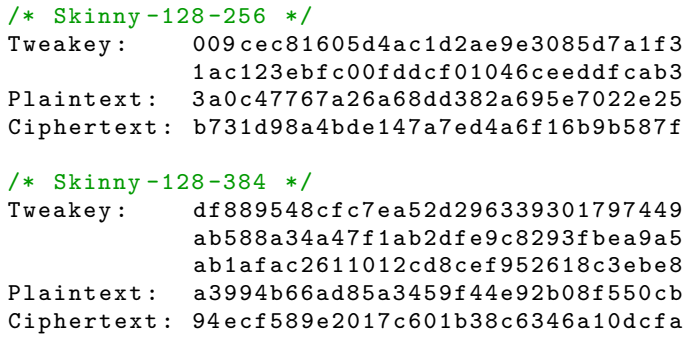




\section{The AEAD Algorithms for M2-M6}

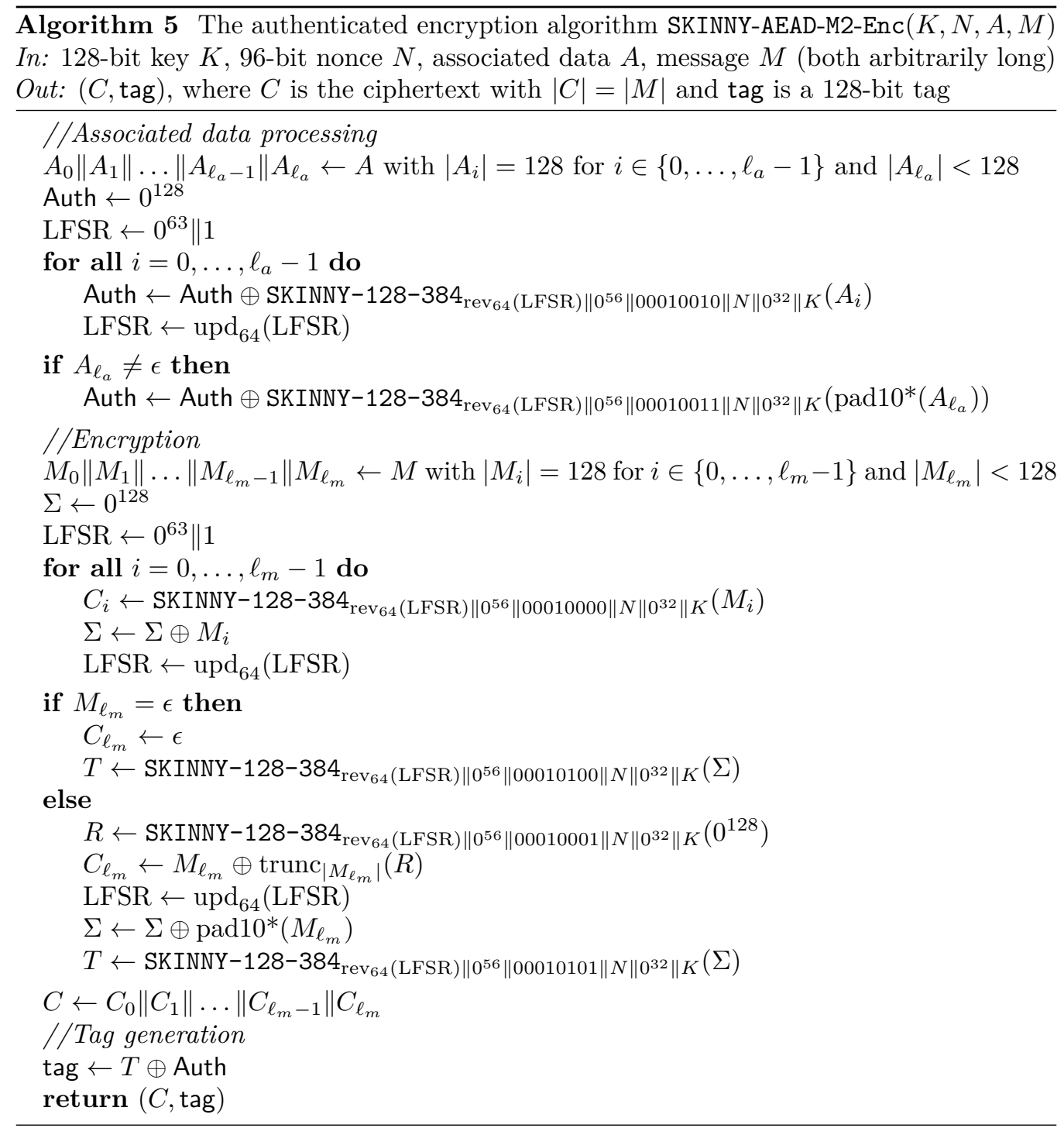




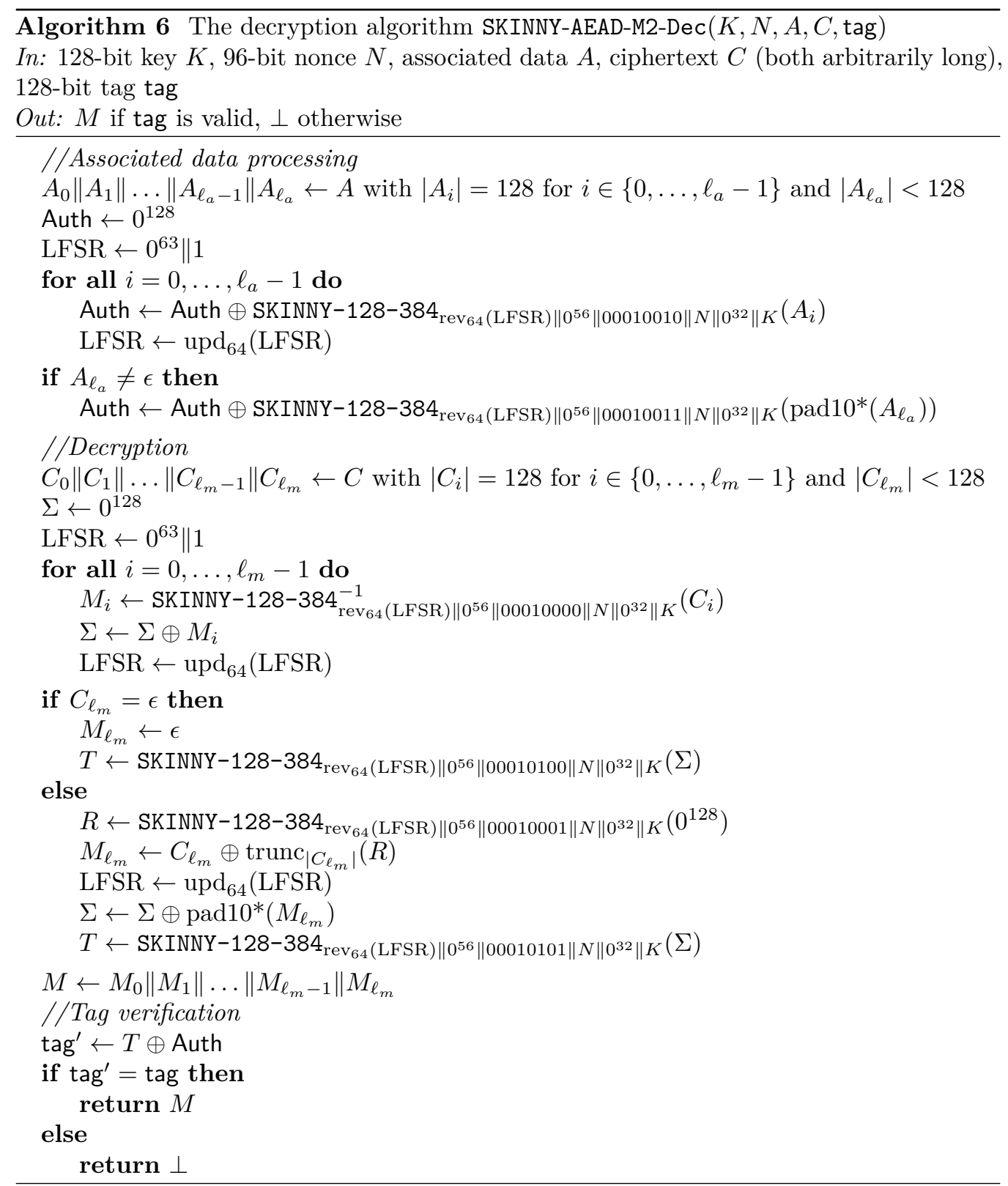




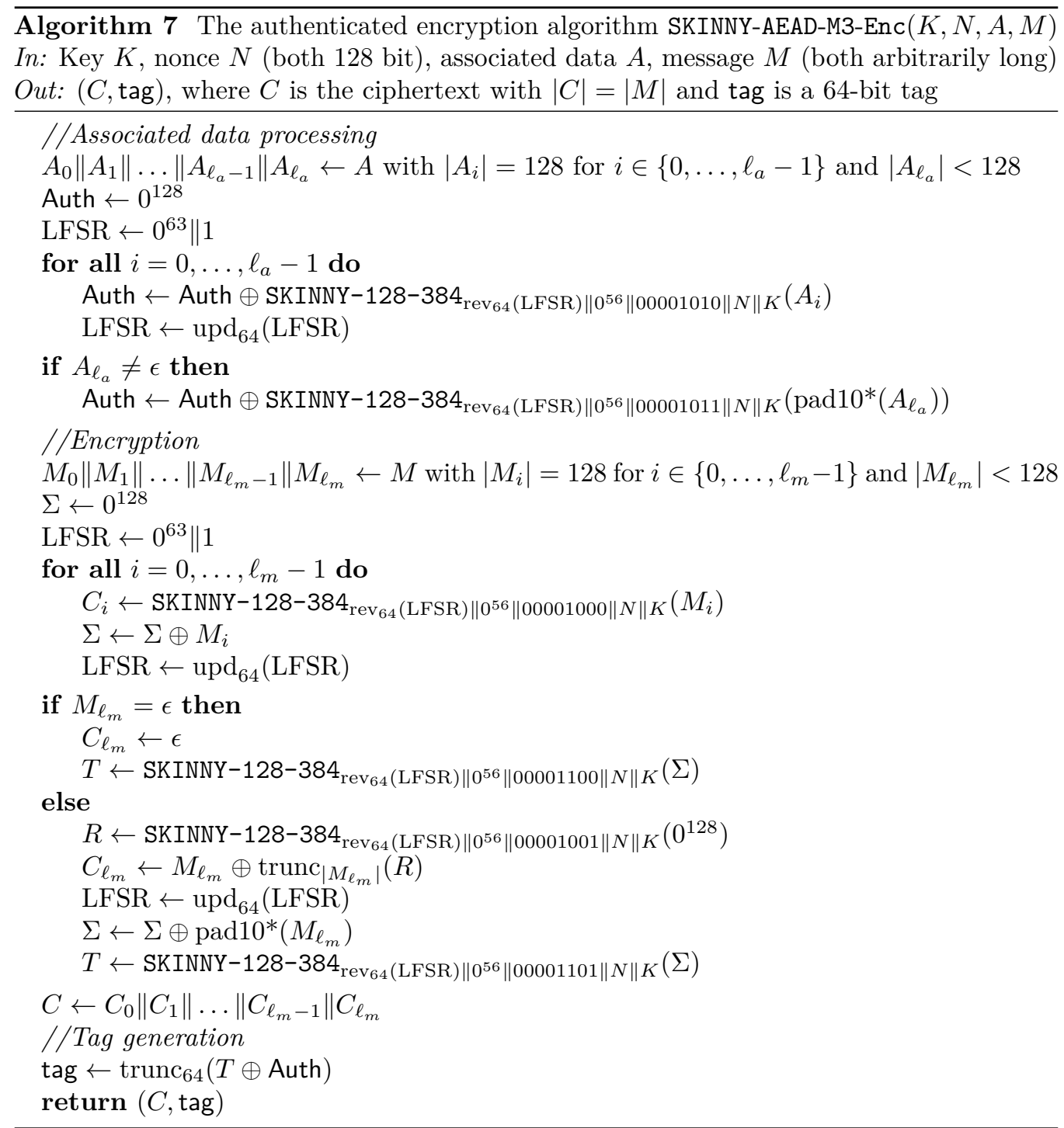




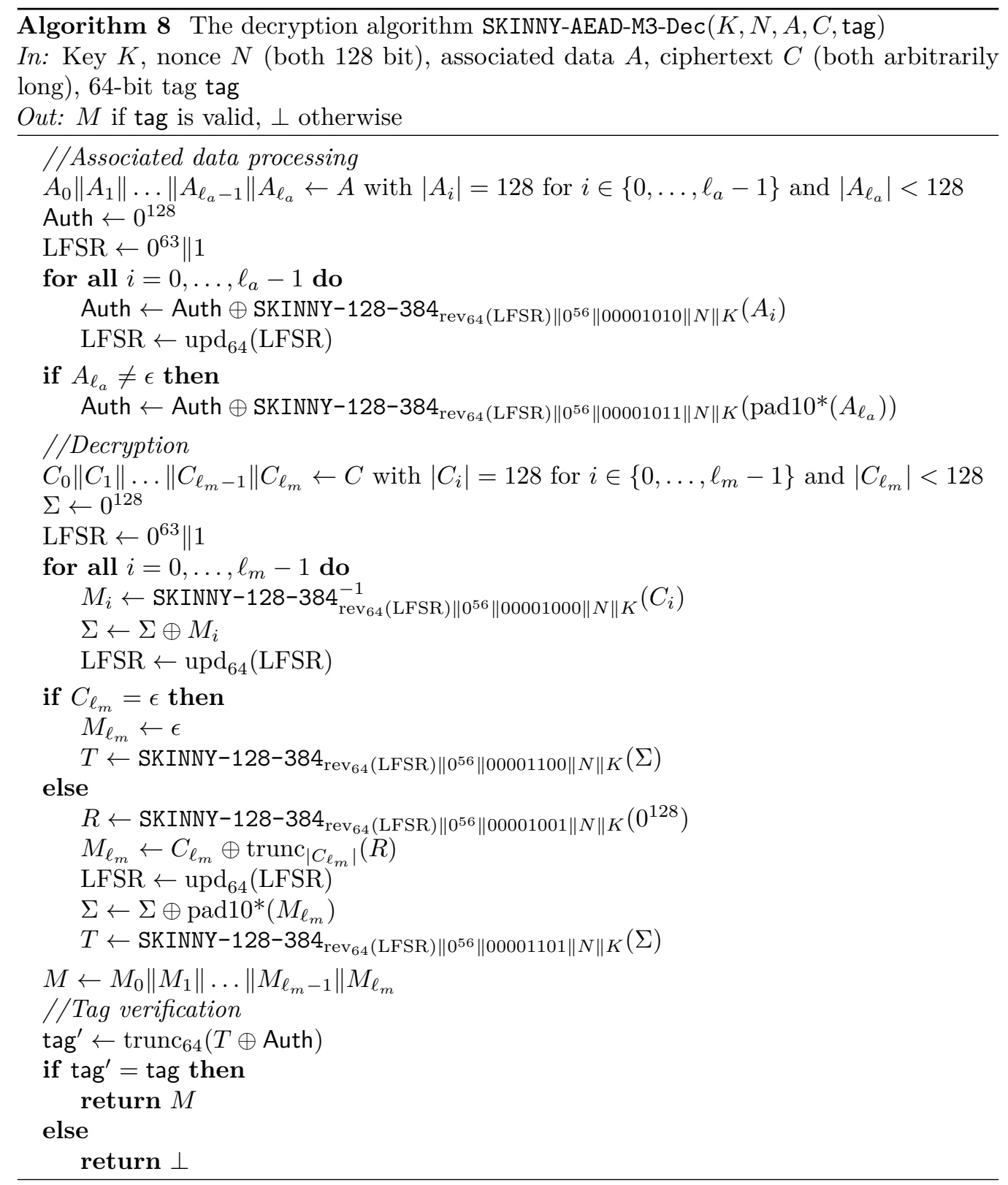




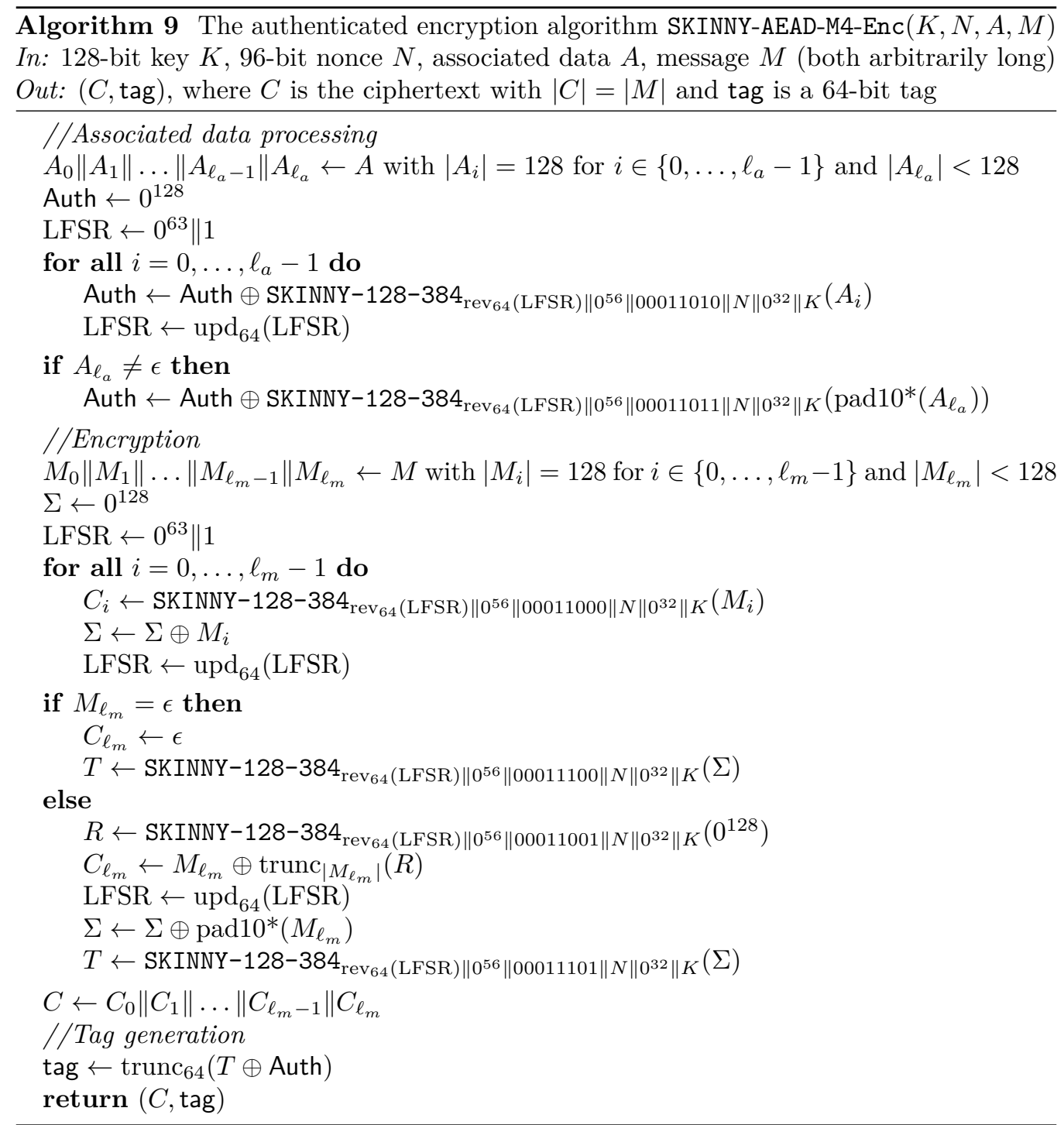




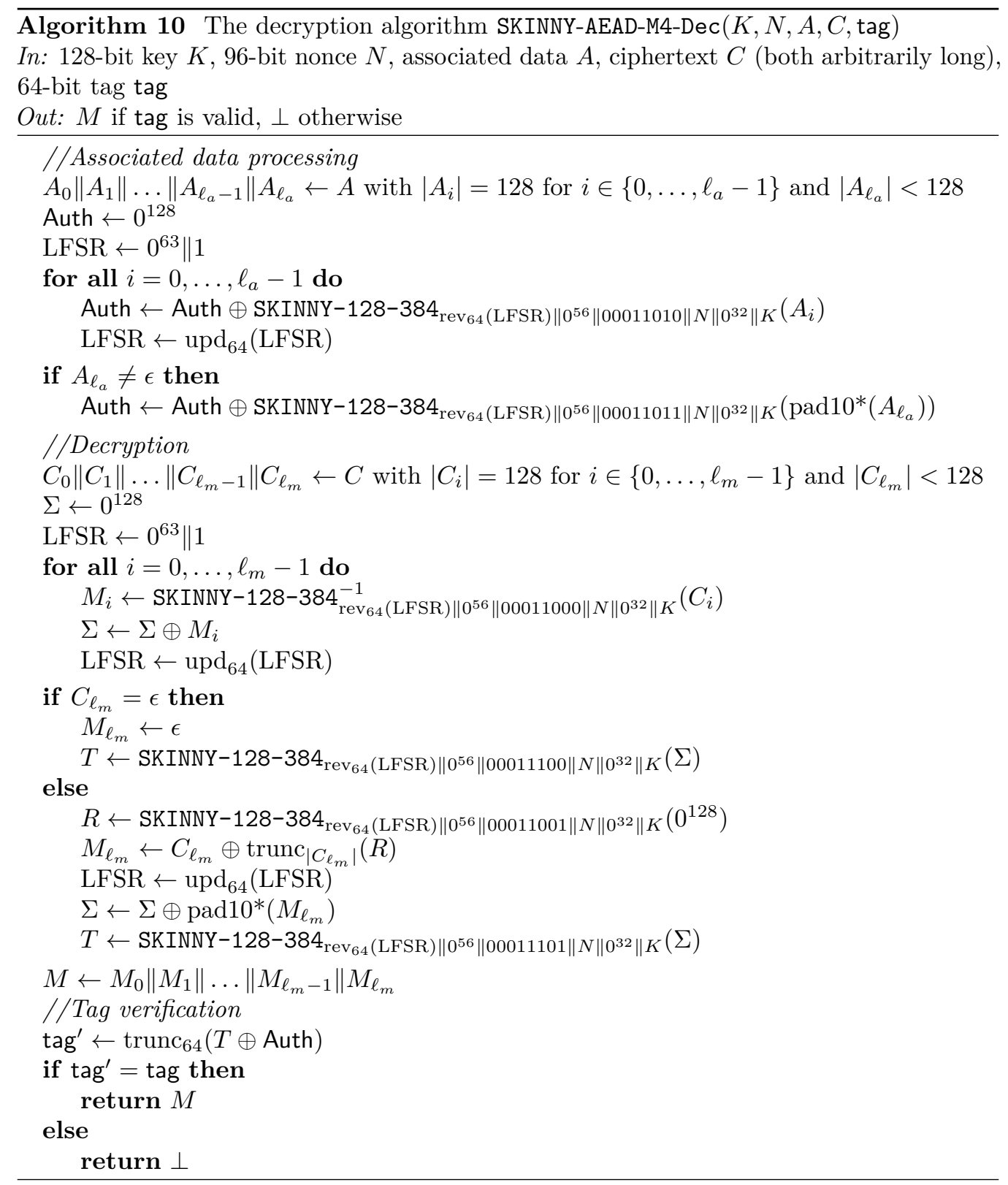




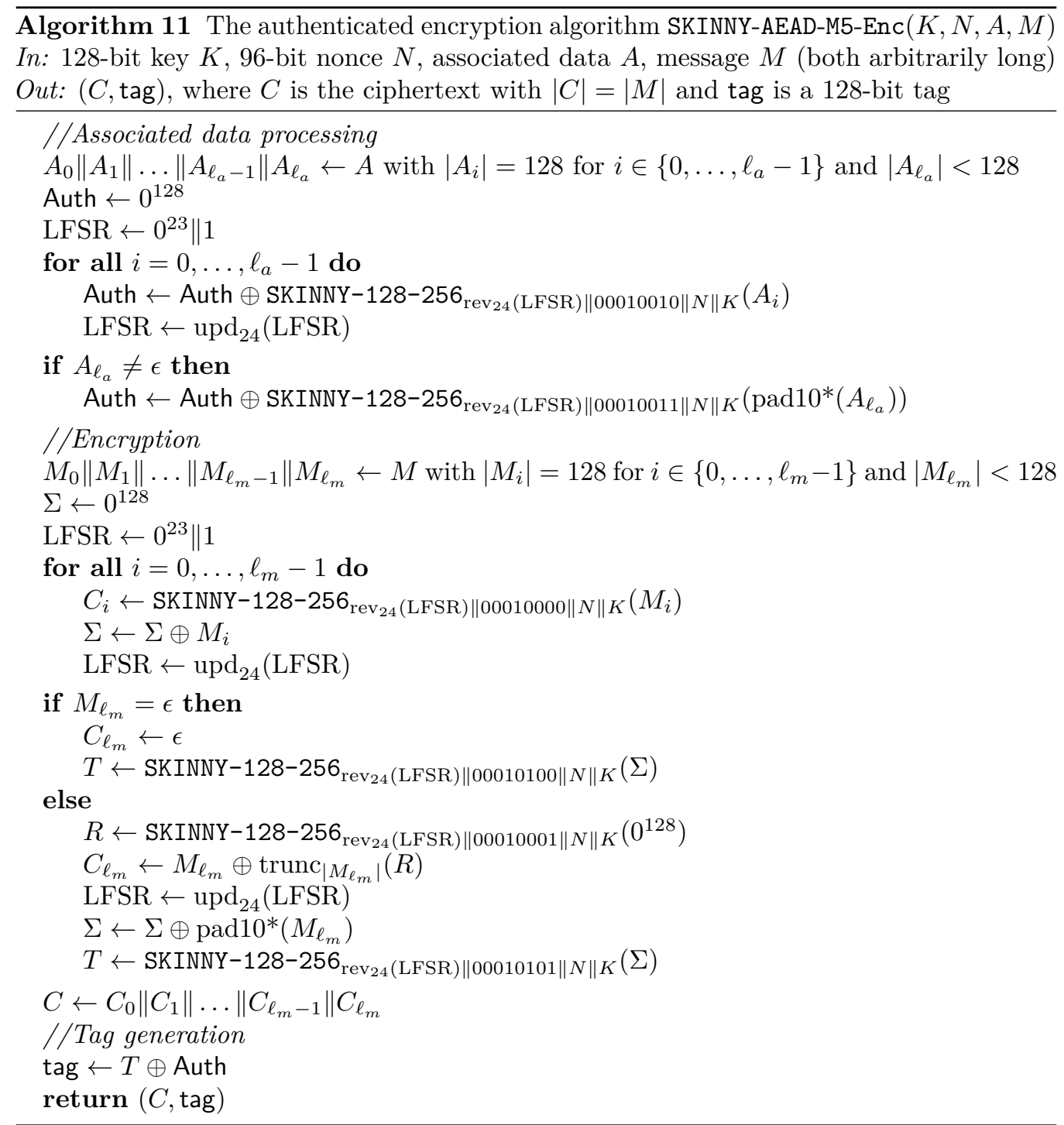




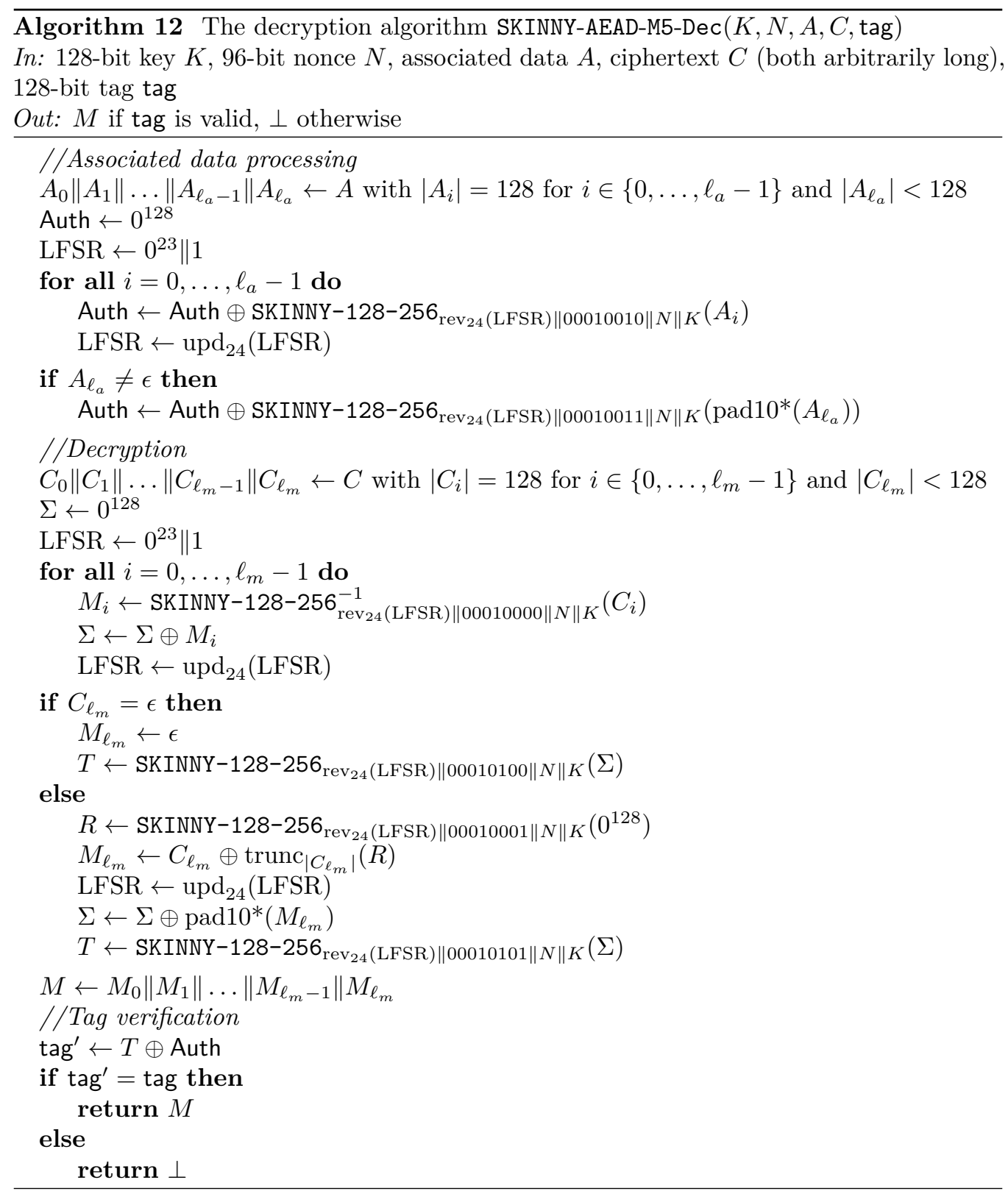




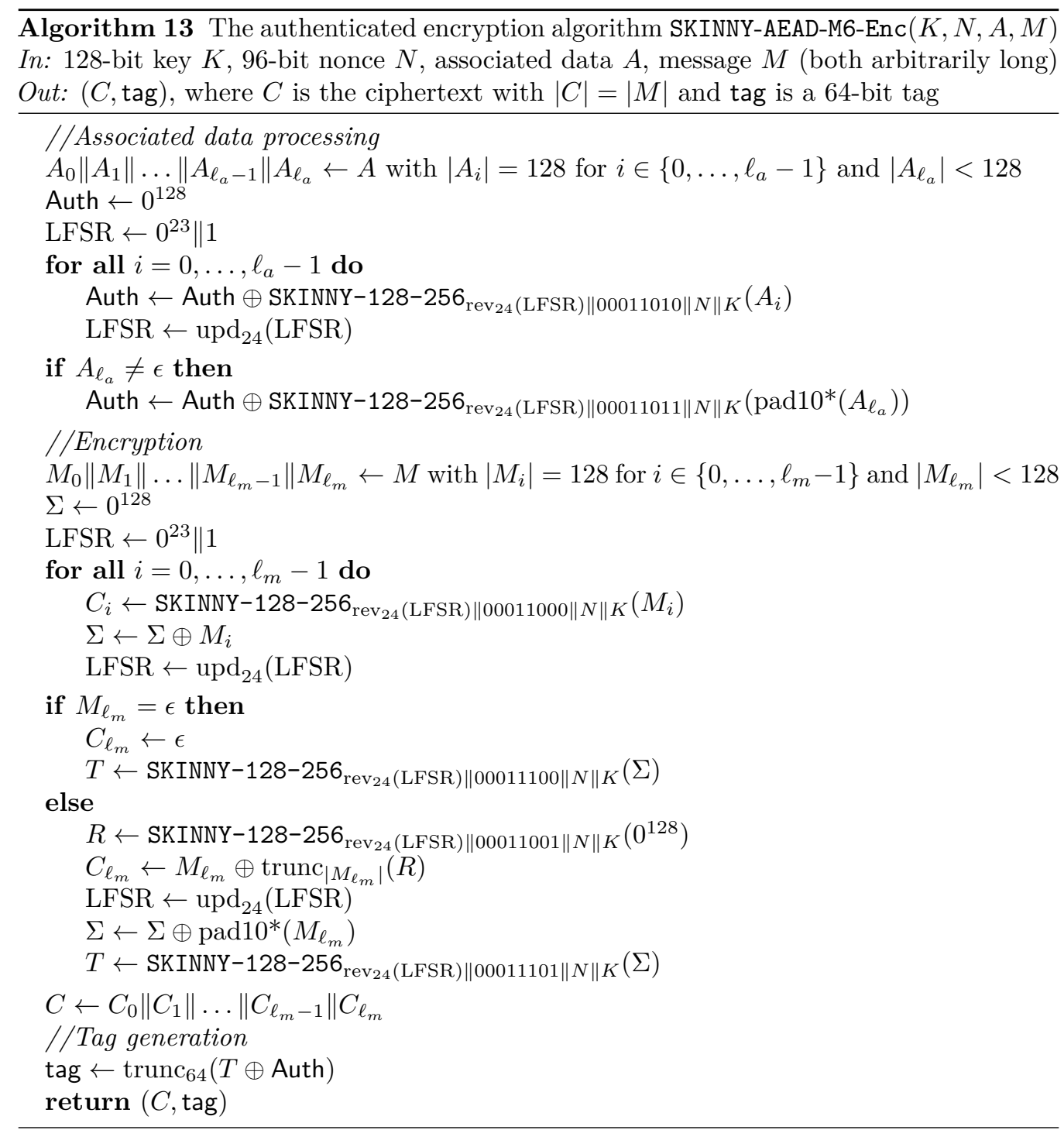




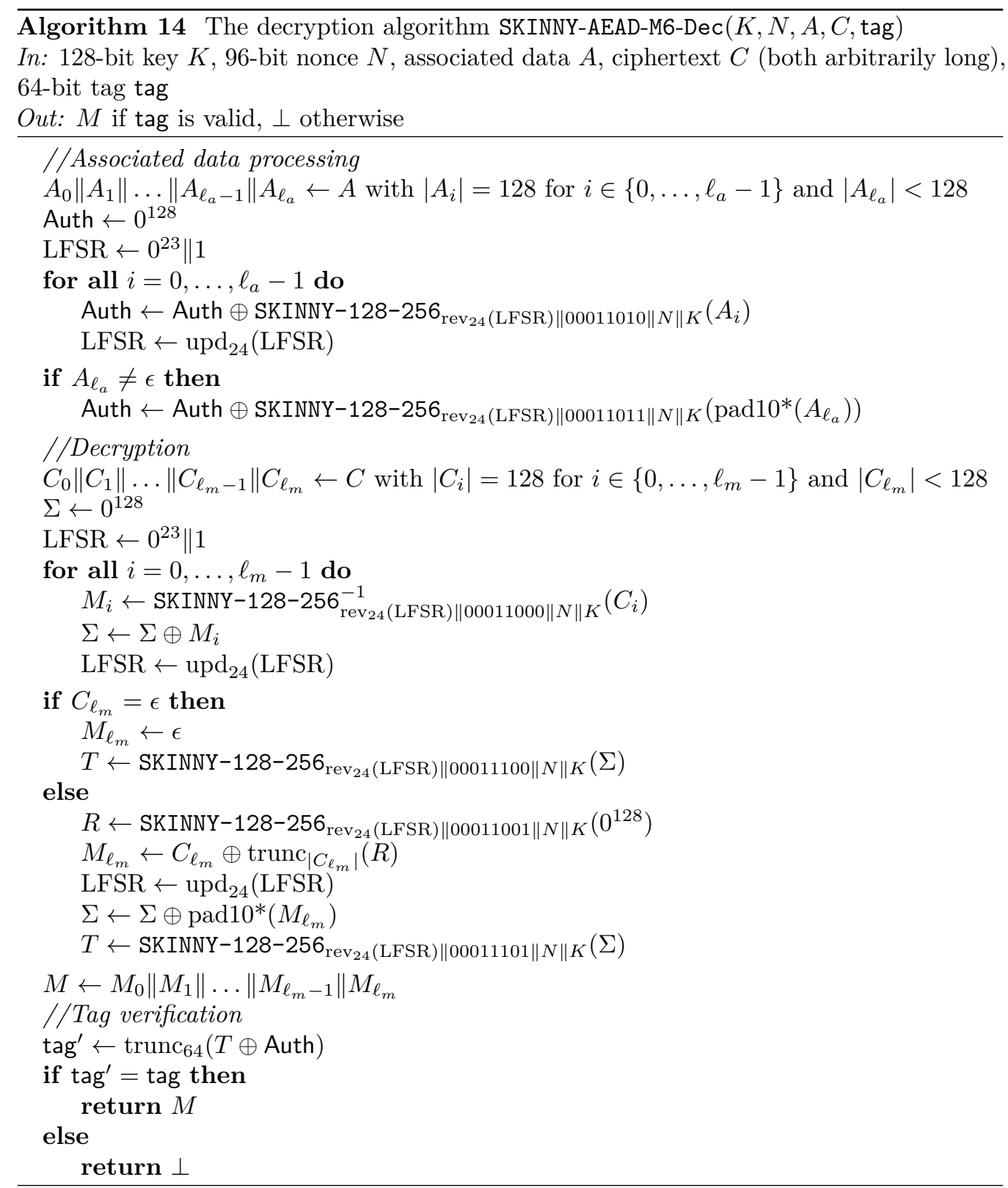

